\title{
Proteomics informed by transcriptomics reveals Hendra virus sensitizes bat cells to TRAIL-mediated apoptosis
}

\author{
James W Wynne ${ }^{1 *}$, Brian J Shiell ${ }^{1}$, Glenn A Marsh', Victoria Boyd ${ }^{1}$, Jennifer A Harper ${ }^{1}$, Kate Heesom²,

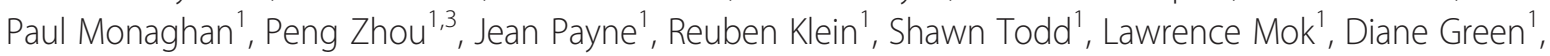 \\ John Bingham', Mary Tachedjian', Michelle L Baker ${ }^{1}$, David Matthews ${ }^{2^{*}}$ and Lin-Fa Wang ${ }^{1,3}$
}

\begin{abstract}
Background: Bats are a major reservoir of emerging infectious viruses. Many of these viruses are highly pathogenic to humans however bats remain asymptomatic. The mechanism by which bats control viral replication is unknown. Here we utilize an integrated approach of proteomics informed by transcriptomics to compare the response of immortalized bat and human cells following infection with the highly pathogenic bat-borne Hendra virus (HeV).

Results: The host response between the cell lines was significantly different at both the mRNA and protein levels. Human cells demonstrated minimal response eight hours post infection, followed by a global suppression of mRNA and protein abundance. Bat cells demonstrated a robust immune response eight hours post infection, which led to the up-regulation of apoptosis pathways, mediated through the tumor necrosis factor-related apoptosis inducing ligand (TRAIL). HeV sensitized bat cells to TRAIL-mediated apoptosis, by up-regulating death receptor transcripts. At 48 and 72 hours post infection, bat cells demonstrated a significant increase in apoptotic cell death.

Conclusions: This is the first study to comprehensively compare the response of bat and human cells to a highly pathogenic zoonotic virus. An early induction of innate immune processes followed by apoptosis of virally infected bat cells highlights the possible involvement of programmed cell death in the host response. Our study shows for the first time a side-by-side high-throughput analysis of a dangerous zoonotic virus in cell lines derived from humans and the natural bat host. This enables a way to search for divergent mechanisms at a molecular level that may influence host pathogenesis.
\end{abstract}

\section{Background}

Emerging infectious diseases pose a significant threat to human and animal welfare. Many emerging and reemerging infectious diseases are zoonoses derived from wildlife, particularly bats $[1,2]$. Bats are now recognized as a major reservoir of zoonotic agents. High profile examples include the henipaviruses (Hendra and Nipah) [3-5], severe acute respiratory syndrome-like coronavirus [6,7], Ebola virus [8] and most recently the Middle East respiratory syndrome coronavirus $[9,10]$. The significance of bats

\footnotetext{
* Correspondence: James.wynne@csiro.au; d.a.matthews@bristol.ac.uk ${ }^{1}$ CSIRO Biosecurity Flagship, Australian Animal Health Laboratory, East Geelong, VIC, Australia

${ }^{2}$ Department of Cellular and Molecular Medicine, School of Medical Sciences, University of Bristol, Bristol, UK

Full list of author information is available at the end of the article
}

as a reservoir for zoonotic viruses was first recognized with the emergence of Hendra virus $(\mathrm{HeV})$ in northern Australia in 1994. In two independent spillover events, $\mathrm{HeV}$ claimed the lives of 15 horses and two humans [3,4]. Approximately four years after $\mathrm{HeV}$ emerged, a related paramyxovirus, designated Nipah virus (NiV), emerged in farmed pigs in Malaysia. Between 1998 and 1999, this virus claimed the lives of 105 humans and resulted in the culling of over one million pigs [5]. NiV outbreaks occur annually in Bangladesh with cases of direct human-tohuman transmission also reported. Bats of the Pteropus genus are the natural reservoir of both $\mathrm{HeV}$ and NiV.

Despite the fact that many of the zoonotic viruses harbored by bats are highly pathogenic to their spillover hosts, bats remain clinically unaffected and rarely display any signs of disease. Some rabies-like viruses are the 
notable exception $[11,12]$. The mechanism by which bats control viral replication remains largely unknown. Despite the absence of clinical disease, bats are capable of shedding virus and triggering subsequent zoonotic transmission. This situation implies bats are capable of controlling viral replication, but not eliminating it. Studies on Ebola have demonstrated that bat lung fibroblasts (derived from the Mexican free-tailed bat) are capable of maintaining a low-level persistent infection with wildtype Ebola Zaire [13]. Recent studies have demonstrated that genes involved in innate immunity have evolved rapidly under positive selection within the Australian black flying fox (Pteropus alecto), indicative of coevolution between virus and host [14]. However, indepth knowledge about the antiviral response of bats is still lacking, as are the reagents necessary to study these non-model species. The present study addresses this knowledge gap by comparing the response of $P$. alecto with humans following $\mathrm{HeV}$ infection. As the natural reservoir of $\mathrm{HeV}, P$. alecto remains clinically asymptomatic. By contrast, zoonotic transmission of $\mathrm{HeV}$ to horses and humans is often fatal [15].

Genomic resources are now available for a number of bat species, including whole draft genome sequences [14,16-18] and de novo assembled transcriptomes $[19,20]$. A draft genome sequence for the P. alecto was released in 2013 [14]. However, to date, no studies have examined the antiviral response of this species - or any other bat species - to infectious viruses at either the transcriptome or proteome level. The study of infectious agents in any non-model organism by high-throughput techniques is severely constrained by the quality and availability of gene model annotations, particularly in the field of proteomics. While the draft $P$. alecto genome was annotated using a combination of homology, de novo prediction and transcriptomics [14], continual refinement is necessary. To circumvent the reliance on high-quality annotation models, we recently developed proteomics informed by transcriptomics (PIT) analysis. This technique collects RNA-sequencing (RNAseq) and quantitative high-throughput proteomics data simultaneously, then uses the transcriptomic data to refine and inform the proteomics analysis. We have previously demonstrated that this combined approach sidesteps the issue of bioinformatics annotation and enables the analysis of any species on a similar footing to humans [21].

Using stable isotope incorporation of amino acids in cell culture (SILAC) and RNAseq transcriptomics, we compared the response of kidney cells derived from human and the Australian black flying fox to $\mathrm{HeV}$ infection at 8 and $24 \mathrm{~h}$. Because of the paucity of well-characterized primary cell lines from the Australian black flying fox, we chose to utilize immortalized cells lines from bats (known as PaKiT03) and humans (HEK293T). Attempts were made to choose cells that were transcriptionally and phenotypically similar. Both cell lines were derived from the kidney, immortalized using viral antigens, and are supportive of $\mathrm{HeV}$ replication.

We found that the response of HEK293T cells was significantly different to PaKiT03 cells. The HEK293T cells showed little transcriptional or proteomic response to $\mathrm{HeV}$ at $8 \mathrm{~h}$ post infection (hpi), followed by a global suppression in protein abundance at 24 hpi. By contrast, PaKiT03 cells initially underwent activation of NF-kappa$\mathrm{B}(\mathrm{NF}-\mathrm{kB})$ at $8 \mathrm{hpi}$, followed by the induction of extrinsic apoptosis pathways at $24 \mathrm{hpi}$. Further analysis of the responses after 48 and $72 \mathrm{~h}$ of $\mathrm{HeV}$ infection revealed that PaKiT03 cells, but not HEK293T cells, underwent apoptotic cell death. In addition, our study provides proteomic evidence for over 5,000 genes in the $P$. alecto genome, including several hundred previously unannotated genes. To our knowledge, this is the first study to examine the transcriptional and proteomic response to $\mathrm{HeV}$ in any cell type. Furthermore, this study represents the first side-byside transcriptomic and proteomic analysis of a single virus in two relevant host species. This study generates valuable knowledge concerning the divergent response of a reservoir and susceptible host to a highly pathogenic bat-borne virus.

\section{Results}

\section{HeV transcription comparison between cell lines}

Virus nucleocapsid $(\mathrm{N})$ protein was detectable in increasing numbers of cells at 8 and 24 hpi (Figure 1A-F). Viralinduced syncytia were observed at $24 \mathrm{hpi}$ in HEK293T cells (Figure 1F), but not in PaKiT03 cells (Figure 1C). RNAseq reads from both cell lines were mapped to the $\mathrm{HeV}$ genome using TopHat. We randomly selected one million mapped reads from PaKiT03 and HEK293T cells at 24 hpi to enable a side-by-side comparison of levels of expression from $\mathrm{HeV}$ genes in each cell line (Figure 1G). Comparing gene expression in the two species cell lines revealed similar numbers of reads mapped against the $\mathrm{N}$ and matrix protein $(\mathrm{M})$, with differences in all the other genes (Figure 1G,H). A steep decline in the abundance of transcripts at the M-F gene boundary was observed in both cell lines.

\section{$\mathrm{HeV}$ induces differential gene and protein expression}

A total of 222,569 and 285,918 transcripts (with a predicted open reading frame (ORF) >200 nucleotides) were assembled de novo for the PaKiT03 and HEK293T cells, respectively. Differential expression analysis of de novo assembled transcripts revealed that the overall response to $\mathrm{HeV}$ was distinct between cell lines (Figure 2A). In the PaKiT03 cells, HeV infection induced the expression of over 200 transcripts at $8 \mathrm{hpi}$ and over 600 transcripts at 24 hpi (Figure 2A, Additional file 1). By contrast, only 8 and 31 transcripts were up-regulated at 8 and 24 hpi in the HEK293T cells (Figure 2A, Additional file 1). In both 

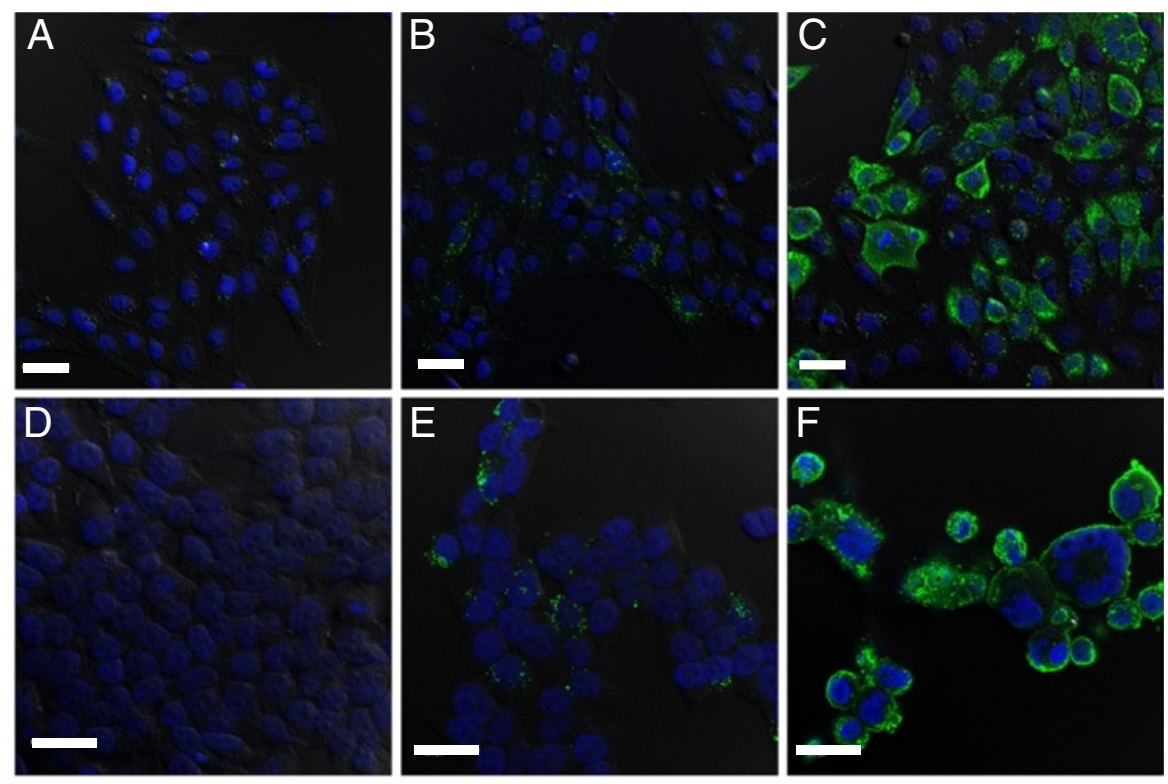

G

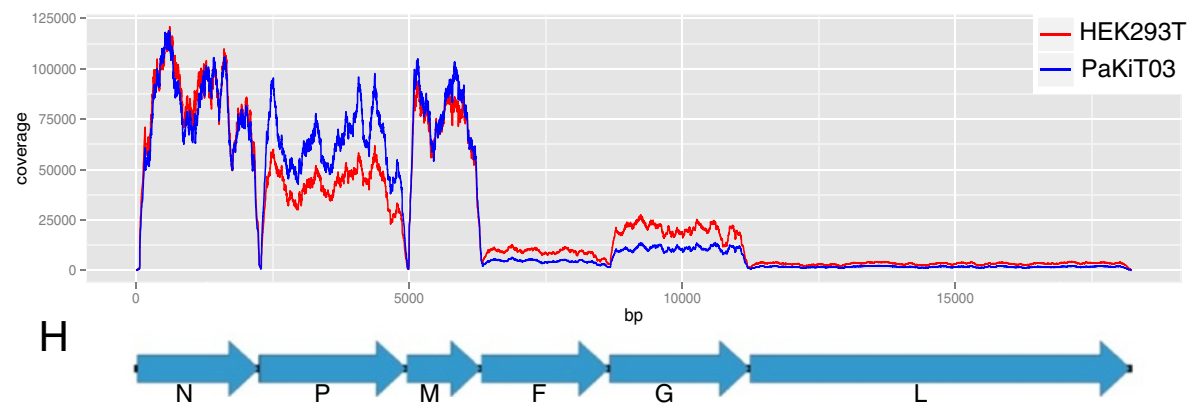

Figure $1 \mathrm{HeV}$ infection and transcription in bat and human cells. Confocal microscopy was used to visualize HeV-infected cells. Nuclei are stained with 4',6-diamidino-2-phenylindole dihydrochloride, and HeV-N protein was immunodetected with an anti-N polyclonal antibody. PaKiT03 cells at (A) 0 hpi, (B) 8 hpi and (C) 24 hpi are shown. HEK293T cells infected with HeV for (D) 0 hpi, (E) 8 hpi and (F) 24 hpi are shown. Scale bar is 30 um. (G) Transcription profile of HeV in PaKiT03 and HEK293T at 24 hpi. (H) Genome structure of HeV. bp, base pairs; F, fusion protein; G, glycoprotein (attachment protein); L, large protein (polymerase); M, matrix protein; N, nucleocapsid; P, phosphoroprotein (includes proteins V, W and C).

cell lines, many of the transcripts induced at 8 hpi remained up-regulated at $24 \mathrm{hpi}$. The proteomic response also differed significantly between the two cell lines (Figure 2B). Most notably, in HEK293T cells over 1,500 proteins were downregulated at $24 \mathrm{hpi}$, whereas only 213 proteins were downregulated in PaKiT03 cells at this time point (Additional file 1). Of the 100 proteins that were significantly induced in PaKiT03 cells at 8 and/or $24 \mathrm{hpi}, 17$ were also significantly up-regulated at the mRNA level (Additional file 1). Many of these proteins have proven roles in innate immunity, including the transcription factor CCAAT/enhancer-binding protein delta (CEBPD), the interferon (IFN)-stimulated gene ISG20, receptors CD40 and CD44, and complement component C3. By contrast, no proteins were up-regulated at both the protein and mRNA level in the HEK293T cells. Furthermore, with one exception in the PaKiT03 cells (SLC12A2), the proteins down-regulated at 8 and/or $24 \mathrm{hpi}$ were not significantly down-regulated at the mRNA level for either cell line. A full list of Trinity transcript and protein expression statistics are provided as Additional files 2 (PaKiT03) and 3 (HEK293T).

Using our PIT-based approach we were able to detect a large number of genes and proteins not currently in the UniProt lists for P. alecto. A total of 263 Trinity-specific proteinGroups were identified, which represent potentially novel or alternative protein-coding genes previously not identified in the $P$. alecto genome. Two notable genes identified by this strategy, that were also differentially expressed, were CEBPD and the gene for tumor necrosis factor (TNF) receptor superfamily 26 (TNFRSF26).

\section{Divergent responses of orthologous transcripts}

Next we identified and compared the expression of orthologous transcripts between the PaKiT03 and HEK293T 

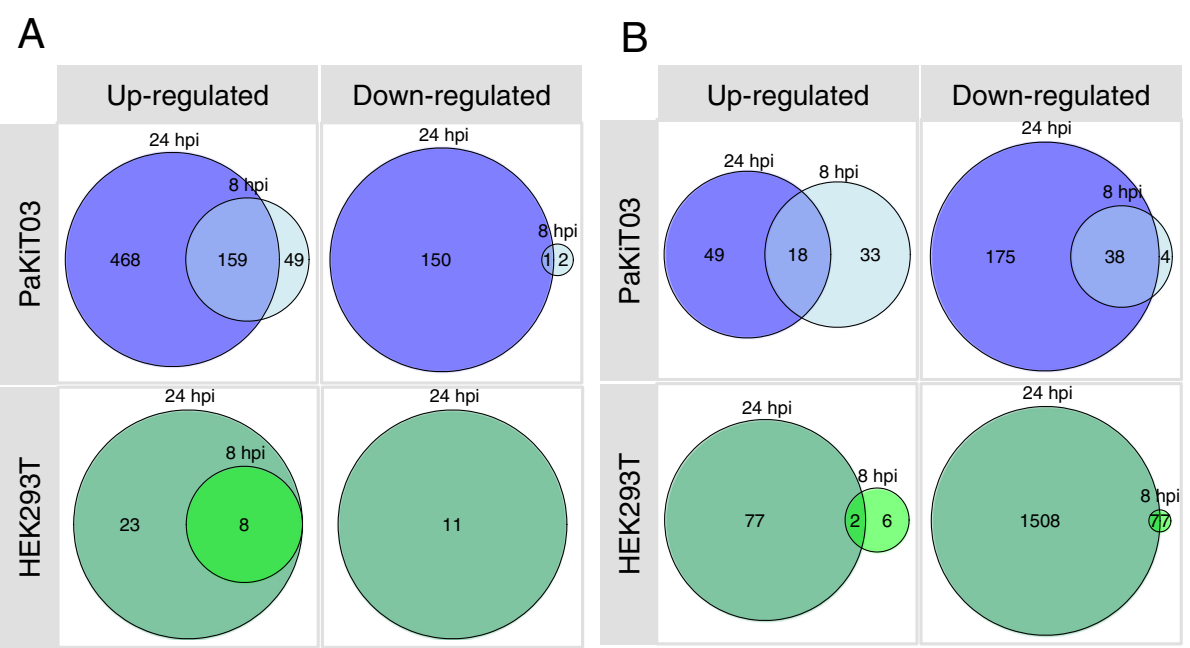

Figure 2 Statistics of differential expression. (A) Differential expression of Trinity transcripts at 8 and 24 hpi was assessed as pairwise comparisons to 0 hpi. The number and overlap of significantly differentially expressed transcripts (adjusted $P<0.05$ ) for 8 and 24 hpi are illustrated as two-way proportional Venn diagrams. Venn diagrams are organized in a $2 \times 2$ matrix where up- and down-regulated statistics are given in the columns and the cell type is given in the rows. (B) Differential expression of Trinity proteins were defined as proteinGroups with SILAC ratios $\geq 2$ fold relative to 0 hpi. Two-way proportional Venn diagrams for proteins differentially expressed at 8 and/or $24 \mathrm{hpi}$ are illustrated as for (A).

cells. We used the RNAseq data to de novo derive transcripts for both cell lines. All PaKiT03 and HEK293T Trinity transcripts with $>70 \%$ similarities to the same human gene were obtained by Basic Local Alignment Search Tool (BLAST). Over 10,000 orthologous transcripts were identified by this strategy (Additional file 4). We first examined the correlation in baseline mRNA expression (at 0 hpi) between the cell lines (Figure 3A). Because SILAC is a relative quantification method we could not compare the baseline protein expression between the cell lines. A Pearson's correlation coefficient of 0.75 demonstrated that orthologous transcripts showed similar baseline mRNA expression prior to $\mathrm{HeV}$ infection. Despite this, at 8 and 24 hpi the correlation in mRNA expression between orthologous transcripts fell significantly (Figure 3B). Similarly, at the protein level there was poor correlation in relative protein abundance of orthologous transcripts at 8 and 24 hpi (Figure 3C). Moderate agreement in mRNA fold change and relative protein abundance was, however, observed between 8 and 24 hpi within each cell line (Figure 3B,C). A heatmap of all orthologous transcripts that were significantly differentially expressed - at one or more time point - confirmed that the response was similar at 8 and 24 hpi within each cell line, but differed between cell lines (Figure 3D).

\section{Enrichment of immune and TNF apoptotic pathways in PaKiT03 cells}

Gene Ontology (GO) enrichment analysis was performed on the combined list of transcripts and proteins that were either up- or down-regulated at one or more time points. Proteins and transcripts that were up-regulated in the
PaKiT03 cells at 8 and/or 24 hpi were broadly involved in response to external stimulus [GO:0009605], response to stress [GO:0006950], defense response [GO:0006952], regulation of apoptotic processes [GO:0042981] and immune system process [GO:0002376] (Figure 4A, Additional file 5). Transcript and protein sets were also subjected to Kyoto Encyclopedia of Genes and Genomes (KEGG) pathway mapping. Many of the up-regulated PaKiT03 transcripts and proteins were mapped to TNF signaling (ko4668), NF$\kappa B$ signaling (ko04064) and cytokine-cytokine receptor interaction (ko04060) pathways (Figure 4B, Additional file 6).

A subset of transcripts involved in apoptosis and TNF signaling (Figure 4C), including TNFRSF10A (DR4), TNF RSF11B, CD40 (also known as TNFR5), CASP13 and TNFAIP3 (A20), were chosen for validation using quantitative PCR (qPCR). In all cases, the differential expression was confirmed, although the magnitude of the fold change was less by qPCR compared to RNAseq (Figure 4D-H). Due to the paucity of bat specific and cross-reactive antibodies, we could only examine the expression of two proteins - CD40 and TNFAIP3 - by Western blotting in the PaKiT03 cells. CD40 was up-regulated over two-fold at the protein level in the SILAC analysis (Figure 4I). No peptides were found for TNFAIP3 within the SILAC analysis, however considering its mRNA was significantly up-regulated, we examined its protein expression with Western blot nonetheless. Both proteins demonstrated differential expression at either 8 and/or $24 \mathrm{hpi}$, similar to their mRNA expression (Figure 4J).

\section{Interferon production and signaling in HEK293T cells}

GO enrichment of HEK293T proteins and transcripts up-regulated at 8 and/or 24 hpi revealed enrichment for 


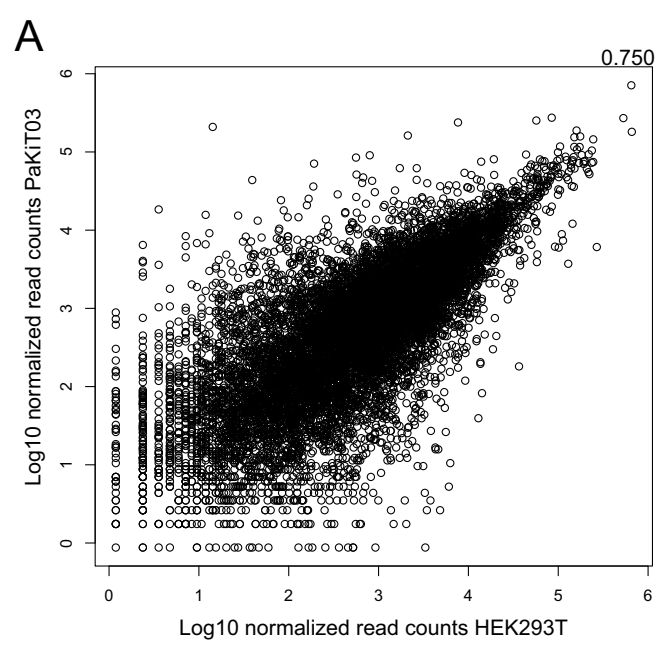

B

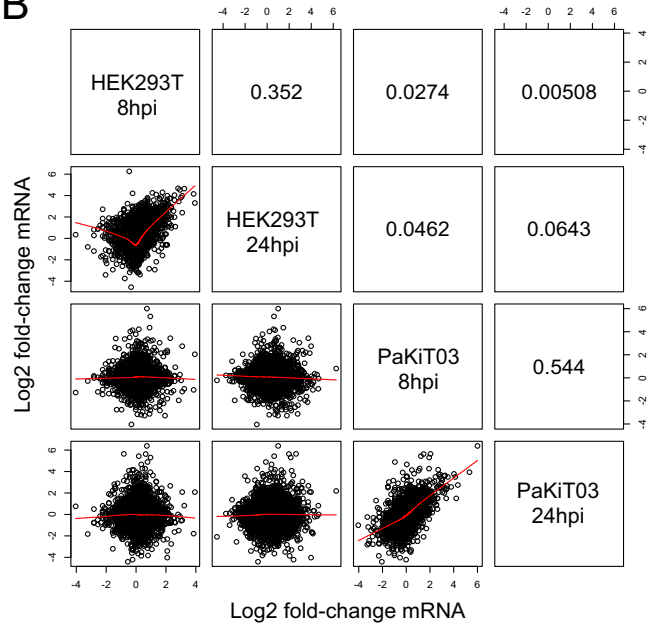

C

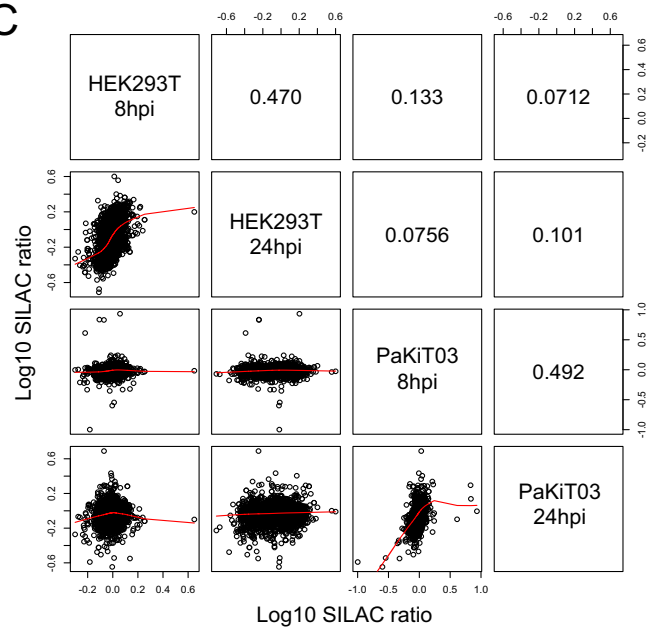

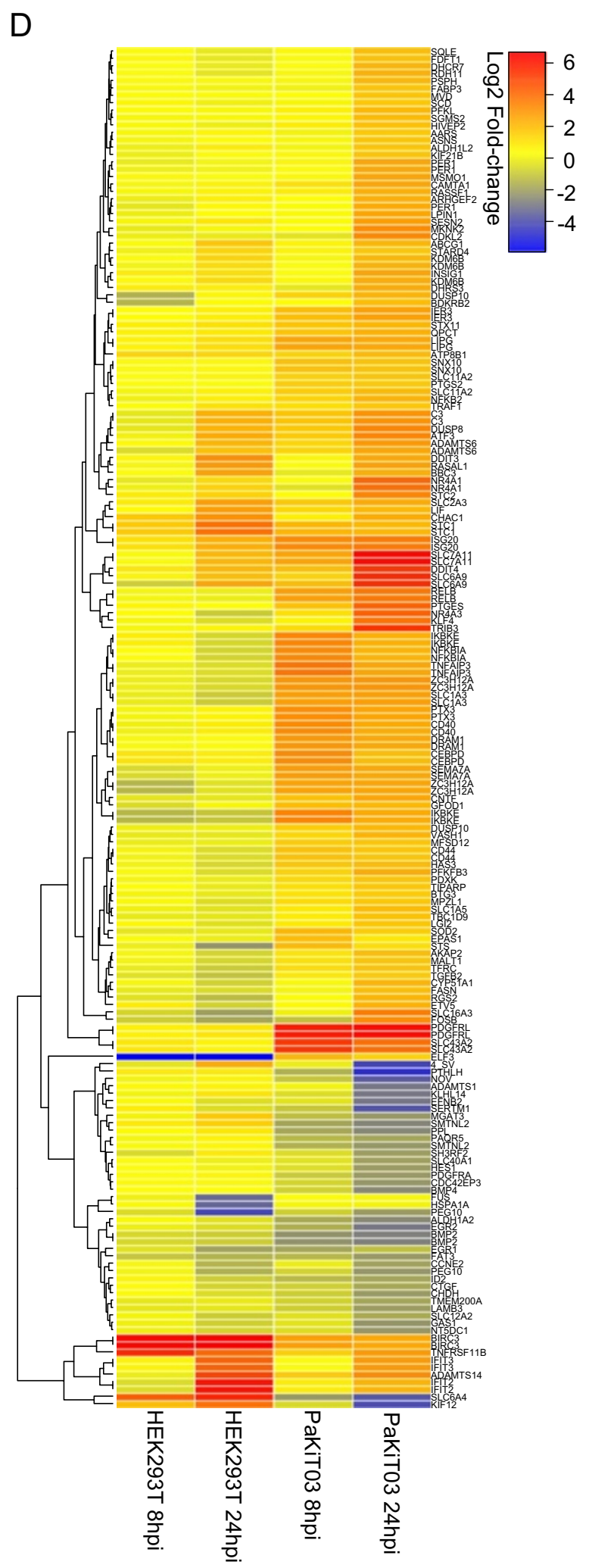

D

Figure $\mathbf{3}$ (See legend on next page.) 
(See figure on previous page.)

Figure 3 Relationship between mRNA and protein expression of orthologous transcripts. (A) Scatterplot illustrating the relationship in baseline (0 hpi) mRNA expression as log10 normalized fragments per kilobase of transcript per million mapped reads (FPKM) for all ortholog transcripts (identity $>70 \%$ ). Pearson's correlation coefficient is indicated in the top right hand corner. (B) Scatterplot matrix of the correlation between transcript expression (log2-fold change) at 8 and 24 hpi in PaKiT03 and HEK293T cells. Pearson's correlation coefficients are given in upper boxes. Simple linear regression models were fitted and are illustrated as red lines through each scatterplot. (C) Scatterplot matrix of the correlation between protein expression (log10 SILAC ratio) at 8 and 24 hpi in PaKiT03 and HEK293T cells. Pearson's correlation coefficients are given in the upper boxes. Simple linear regression models were fitted and are illustrated as red lines through each scatterplot. (D) Heatmap of orthologous transcripts that were significantly differentially expressed at 8 and/or 24 hpi in either cell line. Expression values for transcripts are given as log2-fold change.

only a few biological processes, including response to type I IFN [GO:0034340] and defense response to virus [GO:0051607] (Figure 5A, Additional file 5). Few transcripts and proteins from the HEK293T up-regulated set were mapped to known KEGG pathways, which included the lysosome pathway (ko04142) (Figure 5B, Additional file 6). We validated the expression of three human transcripts (IFNB1, IFIT2 and IFIT3) that contributed to the over-representation of the type I IFN response. All three transcripts demonstrated significant up-regulation at 24 hpi by qPCR (Figure 5C-E).

HeV up-regulates pro-apoptotic pathways in PaKiT03 cells Considering the enrichment of apoptosis-related genes within the PaKiT03 cells, we next examined if the expression of transcripts and proteins involved in the apoptotic response differed between the cell lines. Apoptosis, or programmed cell death, is an important mechanism by which the host can eliminate virus-infected cells. Apoptosis can be triggered through intrinsic (mitochondrial-dependent) or extrinsic signaling pathways. In the present study, a number of genes/proteins involved in the extrinsic TNF receptor-associated inducing ligand (TRAIL)-mediated apoptosis signaling pathway were induced at 24 hpi in the PaKiT03 but not HEK293T cells (Figure 4C). TRAIL-mediated apoptosis occurs through binding of the trimerized ligand to its functional death receptor (DR4/TNFRSF10A or DR5/TNFRSF10B). This is followed by recruitment of the Fas-associated death domain, mediated through a death domain, which induces the sequential activation of caspase 8 (from its pro-caspase precursor) and caspase 3 , ultimately leading to apoptosis-mediated cell death (Figure 6A).

A single peptide derived from the $P$. alecto TRAIL protein was found to be induced over 2-fold at $24 \mathrm{hpi}$ in the PaKiT03 cells (Figure 6B). The fact that TRAIL is often secreted as a soluble form could explain why we only observed a single peptide. This peptide was detected when spectra were searched against the $P$. alecto official UniProt list of proteins and not the Trinity transcriptome-derived protein list. Furthermore, mRNA up-regulation of the functional TRAIL receptor (TNFRSF10A/DR4) was demonstrated by RNAseq and qPCR at 8 and 24 hpi (Figure 4E). In a longer time course experiment we demonstrated that TNFRSF10A/
DR4 mRNA, but not TRAIL, was further induced at 48 and 72 hpi (Figure 6C). A transcript encoding the pro-apoptotic enzyme caspase 13 was also induced at 8 and 24 hpi in the PaKiT03 cells (Figure 4D). Caspase 13 mRNA was also further induced at 48 and $72 \mathrm{hpi}$ in the PaKiT03 cells (Figure 6C). Other genes and proteins with proven roles in intrinsic apoptosis processes, such as bcl 2 binding component $3(B B C 3)$ and baculoviral IAP repeat containing 3 (BIRC3), were also induced in PaKiT03 cells in response to $\mathrm{HeV}$ (Figure 4C).

\section{Inhibitors of apoptosis in PaKiT03 cells}

Interestingly, we also observed up-regulation of the two anti-apoptotic components within $\mathrm{HeV}$-infected PaKiT03 cells, the TRAIL decoy receptor osteoprotegerin (TNFR $S F 11 B$ ) and the ubiquitin ligase A20 (TNFIAP3). TNF $R S F 11 B$ was induced at the mRNA level at both 8 and 24 hpi (Figure 4F). To further examine the role of antiapoptotic components, a long time course experiment was performed on PaKiT03 cells. TNFRSF11B continued to be up-regulated at 48 and $72 \mathrm{hpi}$ (Figure 6C). Acting as a decoy receptor, TNFRSF11B is capable of inhibiting TRAILmediated apoptosis as well as NF- $\mathrm{kB}$ activation. In a similar vein, TNFIAP3 also inhibits TRAIL-induced apoptosis in certain cells, through poly ubiquitination of the receptorinteracting protein 1 (RIP1) and inhibition of caspase 8 cleavage. In this study TNFIAP3 mRNA was significantly induced at 8 and, to a lesser extent, 24 hpi in PaKiT03 cells, but not in HEK293T cells (Figure 4H). Western blot of TNFIAP3 revealed an increase in protein abundance at 8 hpi in bat cells (Figure 4J). TNFIAP3 itself is an NF-kBactivated protein that plays an important role in suppressing NF- $\mathrm{kB}$ activity through a negative feedback mechanism. Again, no differential expression of TNFIAP3 mRNA/protein was observed in the HEK293T cells.

\section{Anti-apoptotic signals in HEK293T cells}

In contrast to the up-regulation of TRAIL and its downstream components in PaKiT03 cells, a down-regulation of proteins involved in both the intrinsic and extrinsic apoptosis pathways was observed in HEK293T cells at 24 hpi. Indeed, the pro-apoptotic proteins $\mathrm{Bcl} 2$ antagonist of cell death (BAD), caspase 2 and TNFR1-associated death domain protein were down-regulated at $24 \mathrm{hpi}$ in HEK293T 


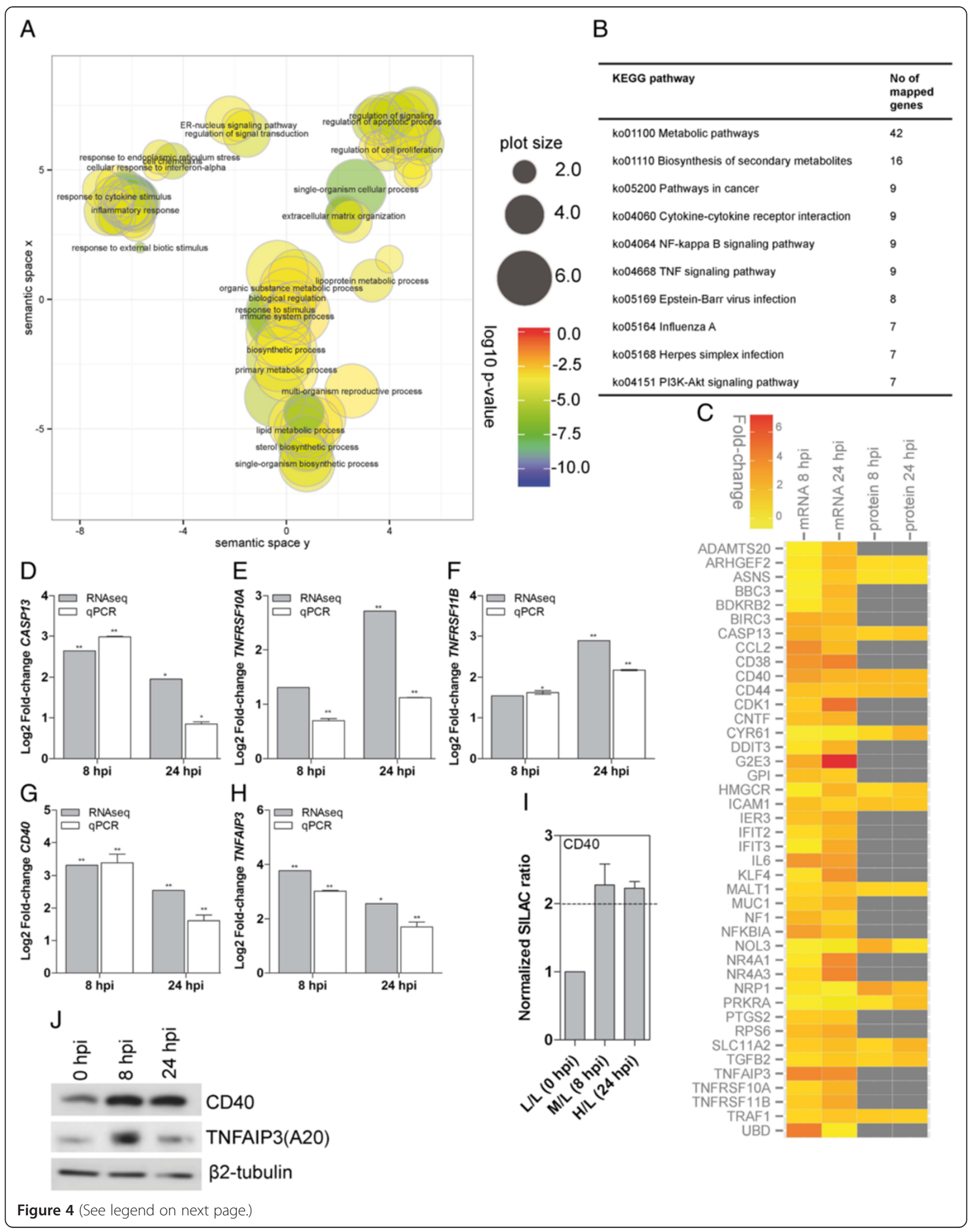


(See figure on previous page.)

Figure 4 Activation of apoptosis pathways in HeV-infected PaKiT03 cells. (A) GO terms enriched in the up-regulated genes/proteins in PaKiT03 cells were analyzed and visualized using REVIGO, where redundant GO terms are removed. The size of the circle represents the number of enriched child GO terms contributing to the parent term. The circle color represents the uncorrected log $10 P$-value for each parent GO term. Semantic space is the result of multi-dimensional scaling where similar GO terms cluster together. A full list of enriched $\mathrm{GO}$ terms, including false discover rate-corrected P-values, are presented in Additional file 5. (B) Top 10 most over-represented KEGG pathways, and the number of significantly differentially expressed genes/proteins contributing to each pathway. The full list of over-represented KEGG pathways is presented in Additional file 6. (C) Expression profile of significantly up-regulated genes/proteins in the PaKiT03 cells which were assigned to the GO term regulation of apoptotic process [GO:0042981] or apoptotic process [GO:0006915]. Gray cells represent proteins where no peptides were observed. Expression values for mRNA are given as log2-fold change, while expression values for proteins are normalized SILAC ratios. Validation of RNAseq differential expression with real-time quantitative PCR (qPCR) for (D) CASP13, (E) TNFRSF10A, (F) TNFRSF11B, (G) CD40 and (H) TNFAIP3/A20 in PaKiT03 cells. Real-time PCR differential expression was calculated based on relative expression to 0 hpi and normalized to GAPDH. Log2-fold change is presented; ${ }^{*} P<0.05$, ${ }^{*} P<0.01$. (I) Relative protein expression profile of CD40 in PaKiT03 cells measured by SILAC. (J) Protein expression profile of CD40 and TNFAIP3/A20 in PaKiT03 cells measured by Western blot. $\beta 2$-tubulin served as the load control.

A

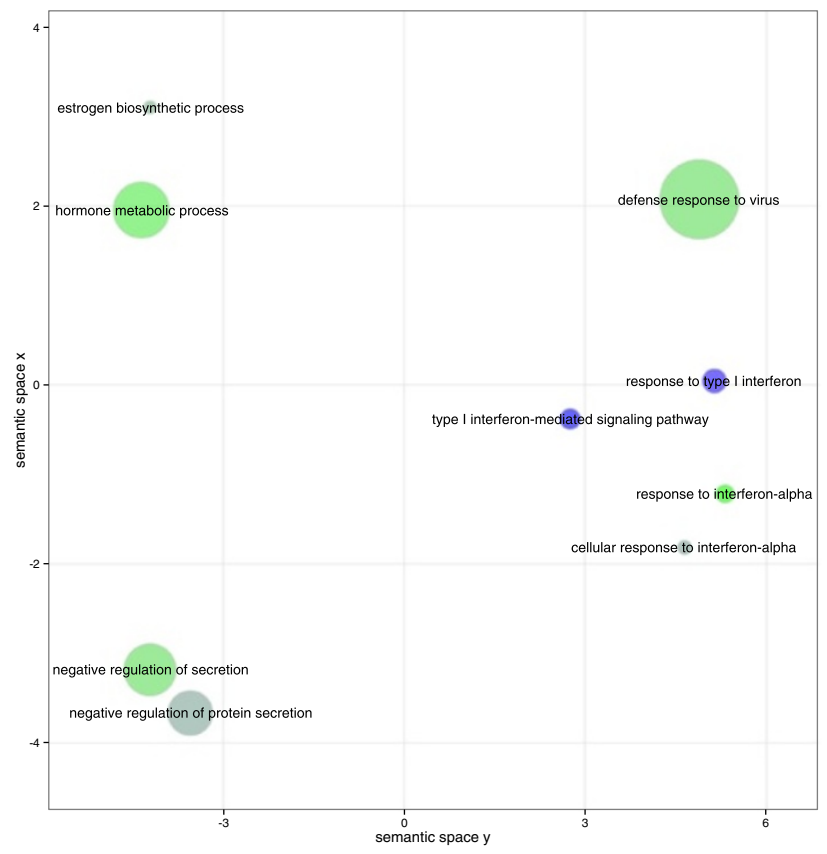

C

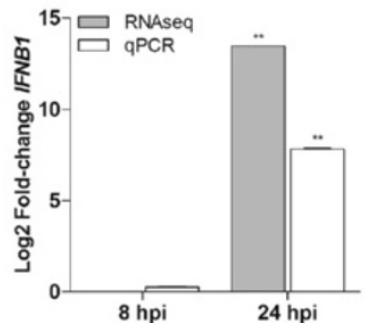

\section{D}

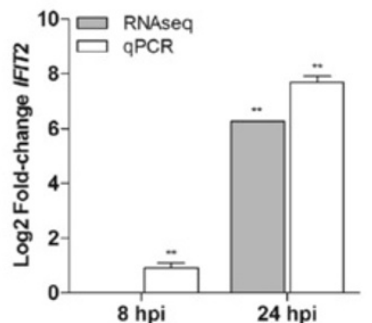

B

\begin{tabular}{ll}
\hline KEGG pathway & $\begin{array}{l}\text { No of } \\
\text { mapped } \\
\text { genes }\end{array}$ \\
\hline ko01100 Metabolic pathways & 5 \\
k004142 Lysosome & 3 \\
ko00140 Steroid hormone biosynthesis & 2 \\
ko00830 Retinol metabolism & 2 \\
ko00330 Arginine and proline metabolism & 1 \\
ko03440 Homologous recombination & 1 \\
ko04612 Antigen processing and presentation & 1 \\
ko00100 Steroid biosynthesis & 1 \\
ko04146 Peroxisome & 1 \\
ko00750 Vitamin B6 metabolism & 1 \\
\hline
\end{tabular}

plot size

2.5
3.0

3.5

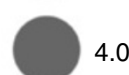

$\bar{\circ}=0.0$

$\frac{\sum^{\frac{1}{C}}}{\frac{1}{(1}}$

$-2.0$

$-3.0$ $-4.0$
E

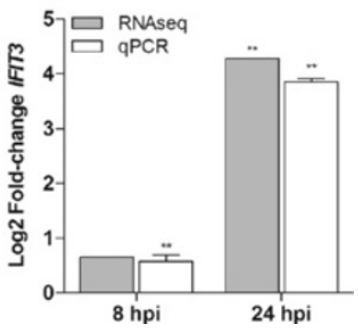

Figure 5 Interferon production and signaling in HeV-infected HEK293T cells. (A) GO terms enriched in the up-regulated genes/proteins in HEK293T cells were analyzed and visualized using REVIGO, where redundant GO terms are removed. The size of the circle represents the number of enriched child GO terms contributing to the parent term. The circle color represents the uncorrected log $10 P$-value for each parent GO term. Semantic space is the result of multi-dimensional scaling where similar GO terms cluster together. A full list of enriched GO terms, including false discover rate-corrected $P$-values are presented in Additional file 5. (B) Top 10 most over-represented KEGG pathways, and the number of significantly differentially expressed genes/proteins contributing to each pathway. The full list of over-represented KEGG pathways is presented in Additional file 6. Validation of RNAseq differential expression with real-time qPCR for (C) IFNB1, (D) IFIT2 and (E) IFIT3. Real-time PCR differential expression was calculated based on relative expression to 0 hpi and normalized to GAPDH. Log2-fold change is presented; ${ }^{*} P<0.05$, ${ }^{* *} P<0.01$. 


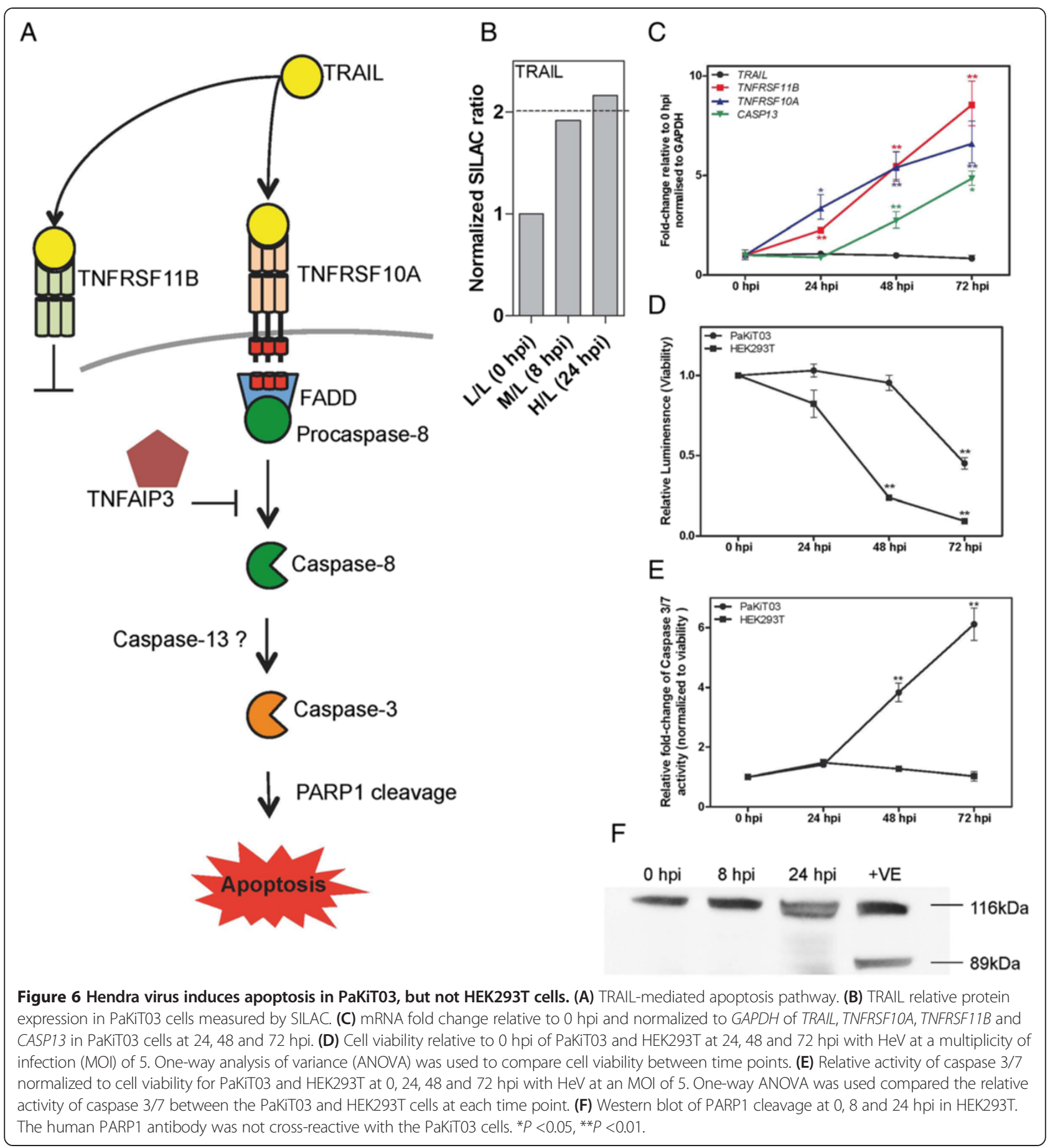

cells (Additional file 1). Interestingly, in the HEK293T cells the TNFRSF11B ortholog was also induced at $24 \mathrm{hpi}$ (although not statistically significantly) (Figure 3D). However, the expression of TNFRSF1OA/DR4 and other functional TRAIL related genes/proteins remained stable in $\mathrm{HeV}$ infected HEK293T cells (data not shown).
$\mathrm{HeV}$ induces apoptotic cell death in PaKiT03 but not HEK293T cells

Considering many apoptotic components (mRNA and proteins) were up-regulated in PaKiT03 but not HEK293T cells, we next set out to test whether $\mathrm{HeV}$ actually induces functional apoptosis in either cell line. Apoptosis was 


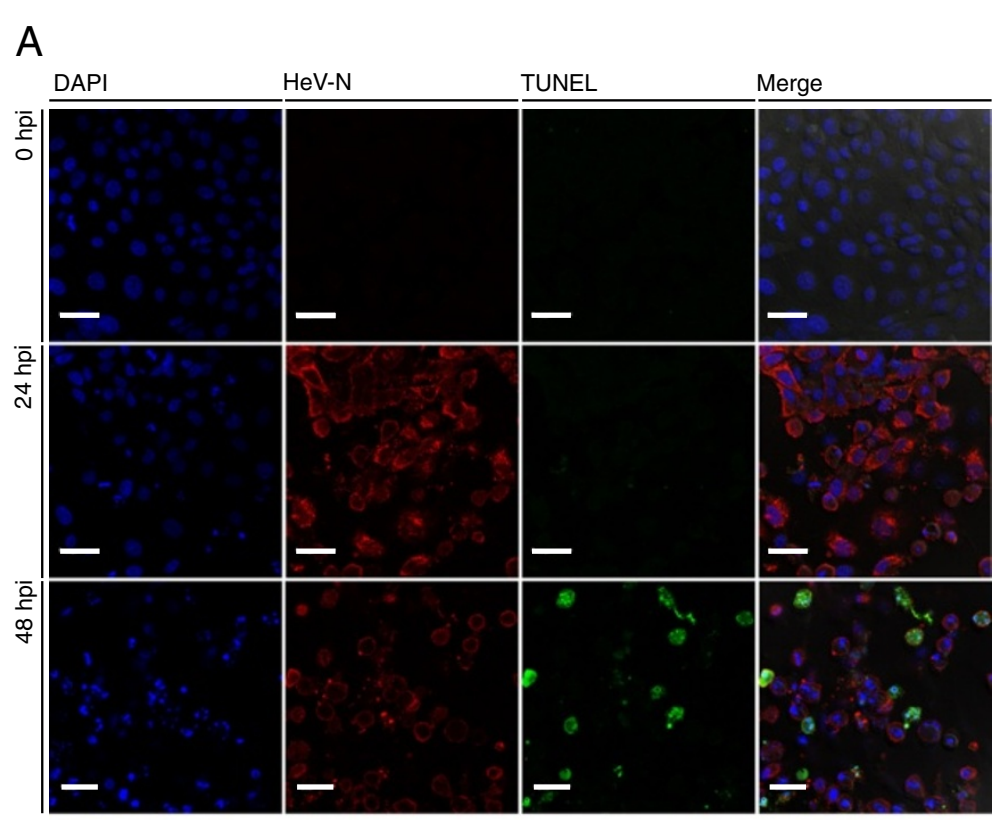

\section{B}

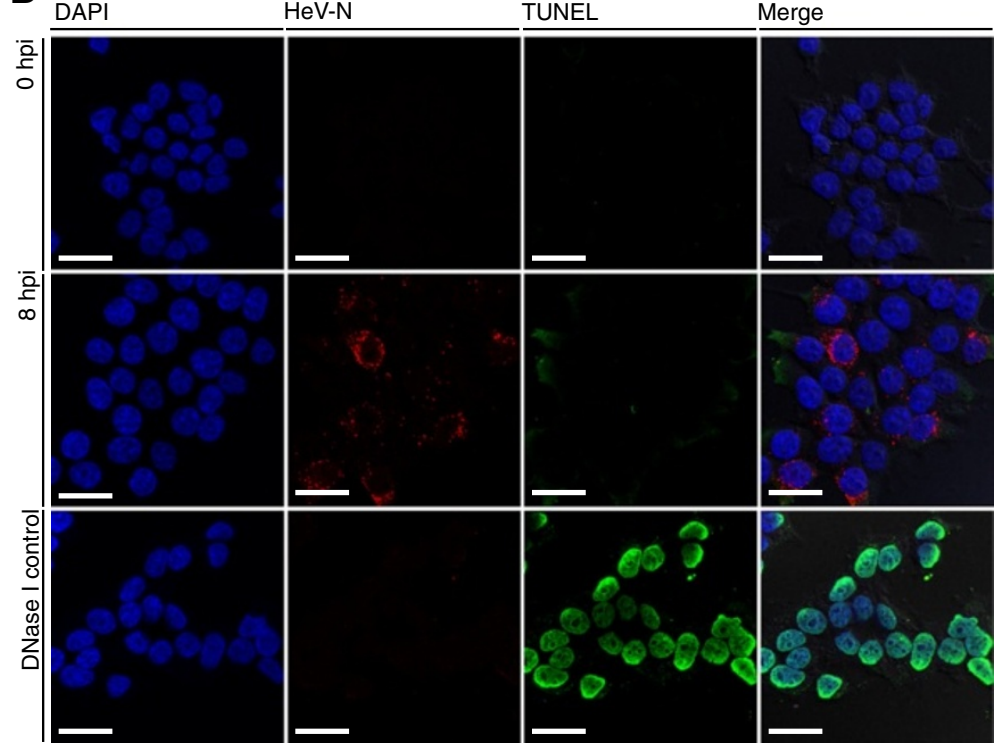

Figure 7 TUNEL staining of HeV-infected (A) PaKiT03 and (B) HEK293T cells. Cells were infected with HeV for either 8, 24 or $48 \mathrm{~h}$ using an $\mathrm{MOI}$ of 5. TUNEL staining was achieved using the Click-iT ${ }^{\oplus}$ TUNEL Alexa Fluor ${ }^{\oplus} 488$ kit (green, fluorescence) and HeV-N was immunodetected as described above (red, fluorescence). DNase I treatment was used as a positive control on HEK293T cells that demonstrated clear nuclear staining. Owing to the decrease in cell viability at $24 \mathrm{hpi}$ and beyond in the HEK293T cells, reliable TUNEL staining could not be performed after $8 \mathrm{hpi}$. Scale bar is $30 \mu \mathrm{m}$ in all panels.

assessed by measuring the activity of the caspase $3 / 7$, the cleavage of PARP1 (HEK293T only) and terminal deoxynucleotidyl transferase dUTP nick end labeling (TUNEL). Both cell lines were infected at a multiplicity of infection (MOI) of 5 for 24, 48 and $72 \mathrm{~h}$ and apoptosis was measured by a Caspase-Glo ${ }^{\circ} / 7$ luminescence assay. Cytopathic effects typical of $\mathrm{HeV}$ were observed after $24 \mathrm{hpi}$ and increased at 72 hpi (data not shown). To account for the decrease in cell viability within infected cells, we first measured cell viability using a CellTiter-Glo ${ }^{\circ}$ luminescence assay and then normalized the caspase 3/7 luminescence to cell viability. As expected, cell viability decreased rapidly after $24 \mathrm{hpi}$ in the HEK293T cells, but not in bat cells. At $24 \mathrm{hpi}$, only a small and non-significant decrease $(P>0.05)$ in the viability of HEK293T cells was observed. At 48 hpi a significant decrease $(P<0.01)$ in cell viability 
was observed in the HEK293T cells, which further decreased at 72 hpi $(P<0.01)$. At 72 hpi the PaKiT03 cells decreased in viability by approximately $50 \%$ (Figure $6 \mathrm{D}$ ).

A significant increase in caspase $3 / 7$ was observed at 48 and $72 \mathrm{hpi}$ in the PaKiT03 compared to uninfected cells $(P<0.01$; Figure 6E). By contrast, no increase in caspase 3/7 was observed in HEK293T cells at any time point. Furthermore, we observed no cleavage of PARP1 in HEK293T cells at 8 or 24 hpi (Figure 6F). The observation that $\mathrm{HeV}$ significantly reduces cell viability of HEK293T cells after 24 hpi meant it was not possible to examine the apoptotic response after this time using either PARP1 Western blotting or TUNEL. TUNEL staining of $\mathrm{HeV}$-infected PaKiT03 cells at 24 and 48 hpi demonstrated an increased number of apoptotic cells at 48 hpi (Figure 7A). This is in agreement with results from the caspase $3 / 7$ assay. In most cases, TUNEL-positive cells were also infected with $\mathrm{HeV}$ at 48 hpi. TUNEL staining of $\mathrm{HeV}$-infected HEK293T could only be performed at 0 and 8 hpi. After this time point $\mathrm{HeV}$ infection caused many cells to detach from the coverslips. TUNEL staining at 0 and 8 hpi revealed no apoptotic HEK293T cells (Figure 7B). This finding is consistent with the lack of PARP1 cleavage at 8 and 24 hpi in HEK293T cells. Weak cytoplasmic staining was observed in some HEK293T cells, but given we observed no nuclear staining (as seen in PaKiT03 and the DNase control) we concluded this was the result of non-specific background staining. The human PARP1 antibody was not crossreactive in the bat cells, and so this method could not be used.

\section{$\mathrm{HeV}$-induced apoptosis in additional human and bat cells}

While a clear difference in $\mathrm{HeV}$-induced apoptosis was observed between the HEK293T and PaKiT03 cells, we concede that these phenotypes may not be representative of all human and bat cells. Given this limitation, we examined whether $\mathrm{HeV}$ induces apoptotic cell death across other human and bat cell types. Owing to the poorly characterized nature and paucity of primary bat cell lines, we concentrated on well-characterized immortalized cell lines from $P$. alecto. Apoptosis was assessed by the Caspase-Glo ${ }^{\circ} / 7$ luminescence assay. Following $\mathrm{HeV}$ infection we observed significant increased caspase $3 / 7$ activities in $P$. alecto fetus cells (PaFeB5) and, to a lesser degree, brain cells (PaBrT03). Activities in both of these cell types were highest at $48 \mathrm{hpi}(P<0.01$, Figure $8 \mathrm{~A}$ ). In contrast to our previous observations in HEK293T cells, the human embryonic fibroblasts (HEF) and HeLa cells demonstrated a strong increase in caspase $3 / 7$ activities at 24 hpi $(P<0.01$; Figure $8 B)$. This response diminished in both HEF and HeLa cells at 48 and 72 hpi. A549 cells demonstrated only a slight but statistically significant increase in caspase $3 / 7$ activity at 48 hpi (p <0.05; Figure 8B).
HeV sensitizes PaKiT03 cells to TRAIL-mediated apoptosis In order to determine the influence of TRAIL in HeVinduced apoptotic death of bat cells, we infected both cell lines with $\mathrm{HeV}$ and simultaneously treated cells with human recombinant TRAIL. At $24 \mathrm{hpi}$, bat cells infected with $\mathrm{HeV}$ and treated with TRAIL had a significant increase in caspase $3 / 7$ activity compared to control cells $(P<0.01$; Figure 9A). Similar observations were made at $48 \mathrm{hpi}$ and, to a lesser extent, $72 \mathrm{hpi}$ in the bat cells. TRAIL alone also significantly increased caspase $3 / 7$ activity at 24 and $72 \mathrm{hpi}$, albeit to a lesser extent compared to TRAIL combined with $\mathrm{HeV}(P<0.01)$. As expected, a significant increase in caspase $3 / 7$ activity was observed in the $\mathrm{HeV}$-infected PaKiT03 cells at 48 and $72 \mathrm{hpi}$ $(P<0.05$; Figure $9 \mathrm{~A})$. By contrast, human cells infected with $\mathrm{HeV}$ and treated with TRAIL showed no increase in caspase $3 / 7$ activity at any time point (data not shown).

Finally, we set out to demonstrate that human (HEK293T) cells are capable of TRAIL-induced apoptotic cell death following viral infection. For this experiment we utilized the bat orthoreovirus Nelson Bay virus (NBV) [22]. NBV is also known as Pteropine orthoreovirus NB (PRV1NB) [23]. Previous studies have demonstrated that mammalian reoviruses can induce TRAIL-mediated cell death in HEK293T cells. HEK293T cells were infected with NBV at an MOI of 5 and treated with human recombinant TRAIL for 24 hpi. A significant increase in caspase 3/7 activity was observed in these human cells simultaneously infected with NBV and treated with TRAIL compared to human cells infected with NBV and treated with TRAIL alone $(P<0.01$; Figure $9 \mathrm{~B})$.

\section{Up-regulation of TRAIL in HeV-infected bat lungs}

To determine whether TRAIL and CD4O mRNA is induced during $\mathrm{HeV}$ infection in vivo, we utilized previously collected tissue samples from an experimental infection of the Australian black flying fox. Kidney and lung tissue samples from four experimental $\mathrm{HeV}$ infected and two naïve control bats were obtained from previous, unpublished studies. Bats were infected with $\mathrm{HeV}$ for $36(\mathrm{n}=2)$ and 60 hours $(\mathrm{n}=2)$. Compared to uninfected animals we observed no increase in CD40 mRNA expression at either 36 or $60 \mathrm{hpi}$ in either the lung or kidney samples (Figure 10A,B). By contrast, TRAIL mRNA was found to be up-regulated approximately five-fold in the lung at 60 hpi (Figure 10B). Because of the biological variation between bats, this increase was not statistically significant. TRAIL mRNA was not induced in the lung at $36 \mathrm{hpi}$ or the kidney at either 36 or 60 hpi.

\section{Apoptotic cell death in vivo}

Given our previous results in vitro, we next set out to investigate whether $\mathrm{HeV}$ could induce apoptosis in vivo 


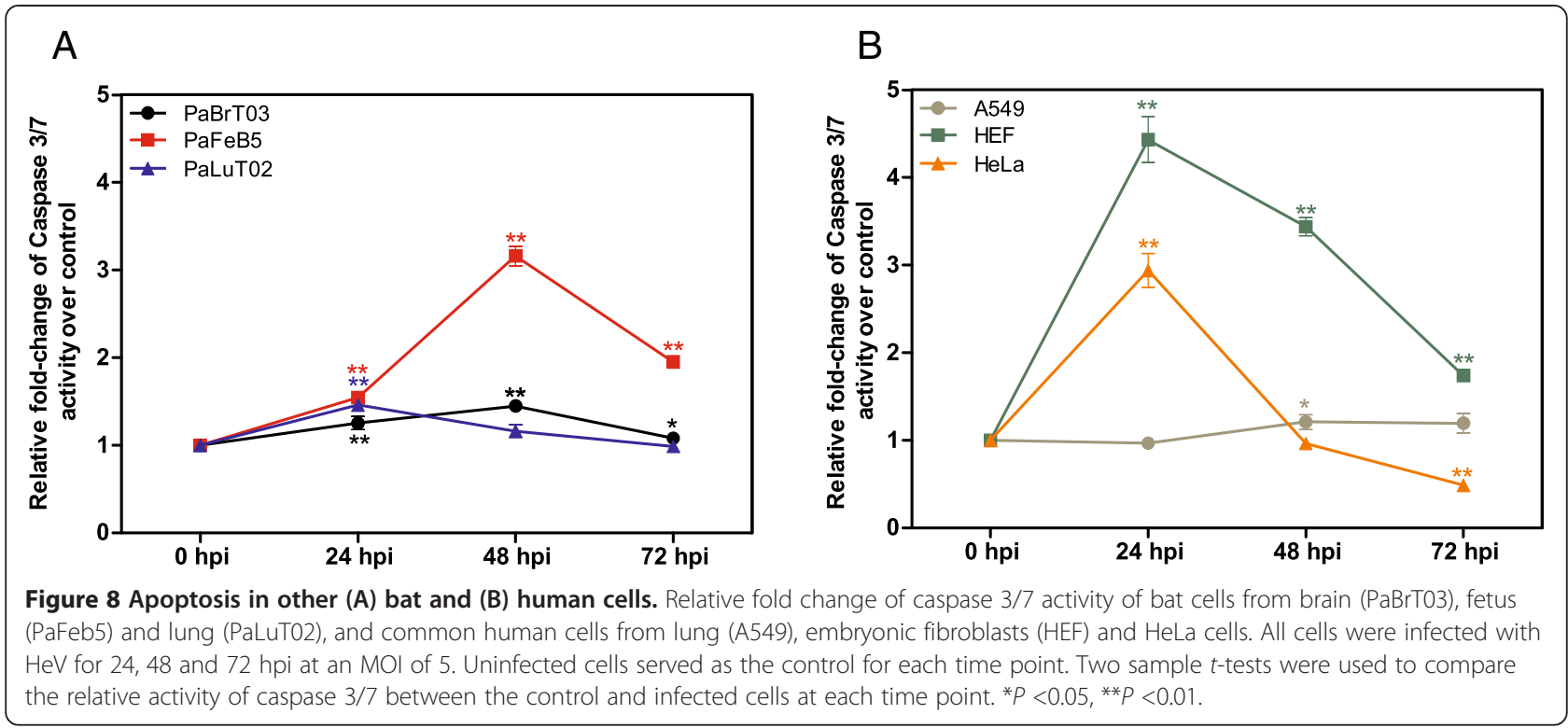

within its natural host, the Australian black flying fox. Formalin-fixed paraffin-embedded tissue sections from the experimental $\mathrm{HeV}$-infected and two naïve control flying foxes were obtained as described above. Colorimetric TUNEL staining was performed on spleen and kidney sections from all bats. Unfortunately the lung sections from this trial were not suitable for TUNEL staining, due to artifacts created during euthanasia. Few apoptotic cells were observed in the kidney in either the $\mathrm{HeV}$-infected (Figure 10C) or control bats (Figure 10D). By contrast, we observed numerous apoptotic cells in the spleen (Figure 10E,F). The number of apoptotic cells within the spleen, however, did not appear to be greater in the $\mathrm{HeV}$-infected bats compared to the naïve controls.

\section{Discussion}

The clinical outcome of humans following $\mathrm{HeV}$ infection is significantly different to that of bats. The ability of bats to maintain asymptomatic viral infections whilst the same agent often causes fatal infections in human is a typical feature of bat-borne zoonotic viruses. The molecular mechanisms responsible for this dichotomy remain unknown. Despite the absence of clinical disease, the fact that bats can shed and transmit viruses suggests bats are capable of controlling viruses, but not eliminating them all together. Rapid induction of the innate immune processes has been proposed as one possible mechanism for subduing viral replication in bats [19]. Considering the importance of bats as natural reservoirs for many emerging viruses, elucidating
A

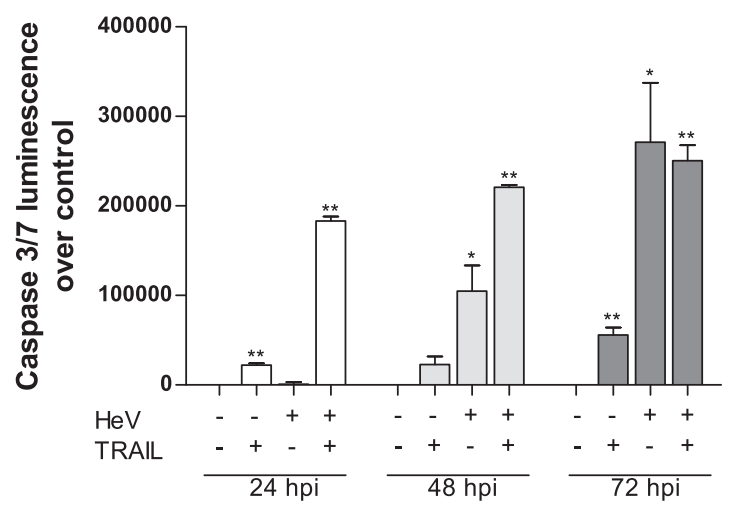

B

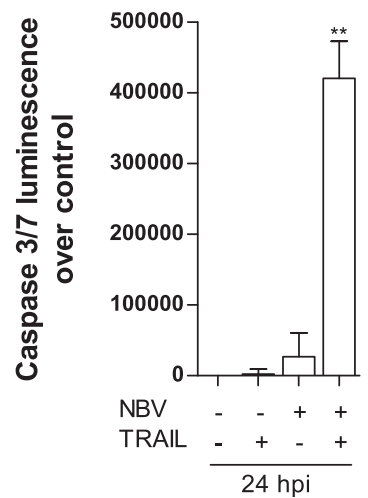

Figure $9 \mathrm{HeV}$ sensitizes PaKiT03 cells to TRAIL-mediated apoptosis. Cells were either treated with $500 \mathrm{ng} / \mathrm{ml}$ human recombinant TRAll and/or infected with virus $(\mathrm{MOI}=5)$ for 24,48 and 72 hpi. The activities of caspase $3 / 7$ within the experimental samples were compared to the control (no TRAlL or virus) using one-way analysis of variance for each time point. ${ }^{*} P<0.05,{ }^{*} P<0.01$. All assays were performed in triplicate. (A) Increase of caspase 3/7 activity over control in PaKiT03 cells following stimulation with human recombinant TRAIL and/or HeV. (B) Increase of caspase 3/7 activity over control in HEK293T cells following stimulation with human recombinant TRAIL and/or Nelson Bay virus (NBV). 
A
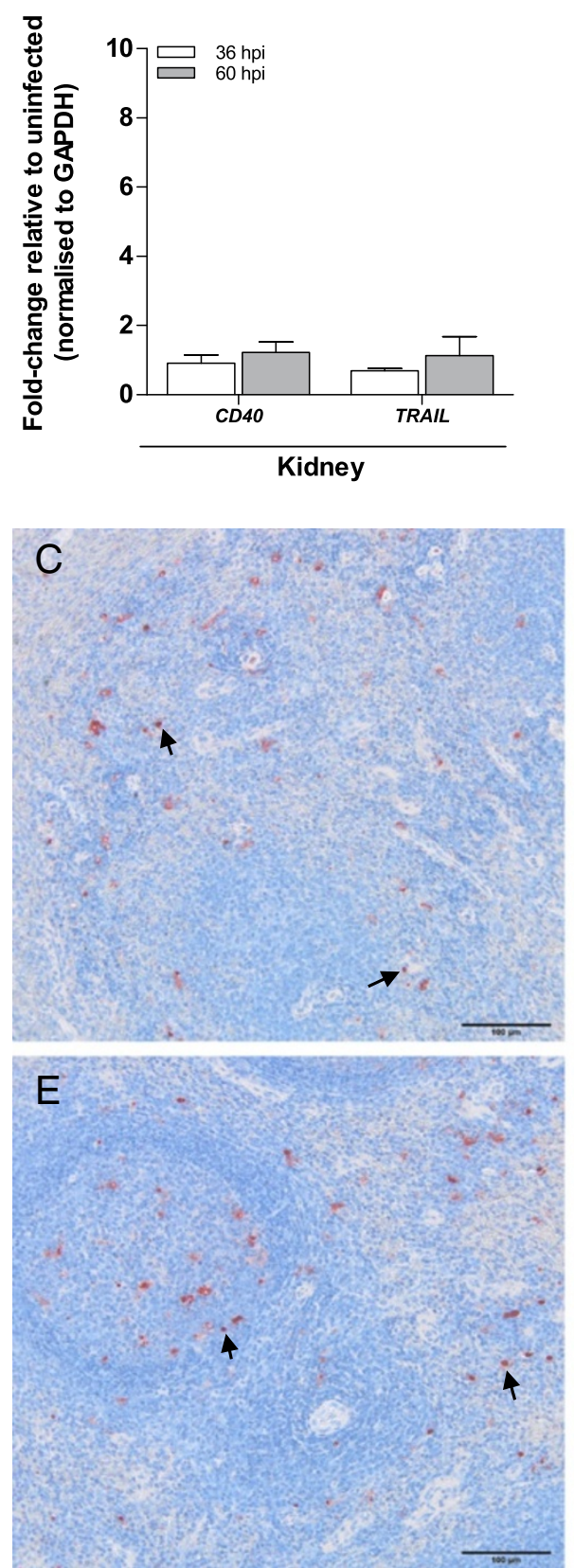

B
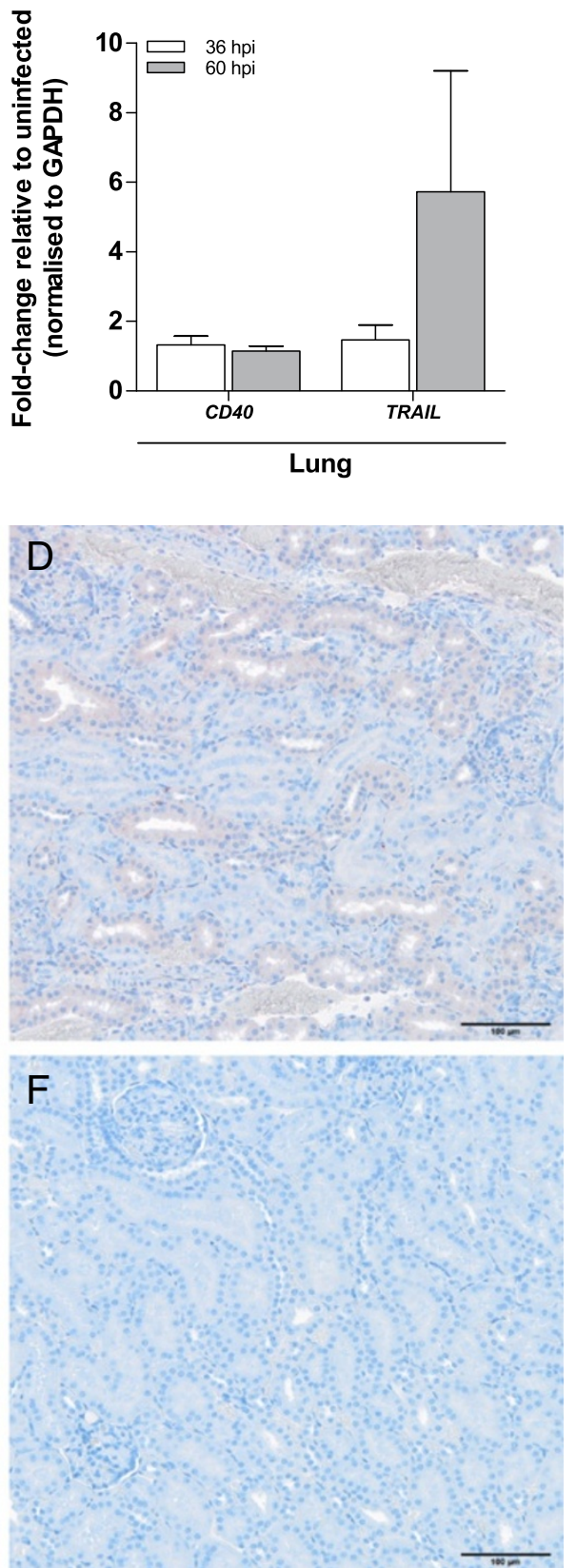

Figure 10 TRAIL expression and apoptosis in bats in vivo. Tissue samples were obtained from a previous unpublished HeV infection of $P$. alecto bats (Australian black flying fox). The expression of CD40 and TRAIL mRNA was compared in (A) kidney and (B) lung and from bats infected with HeV for 36 and 60 hpi compared to uninfected bats. TUNEL staining was also performed on spleen and kidney sections from the experimentally infected and control bats. TUNEL staining of the (C) spleen and (D) kidney of a representative control bat. Arrows highlight TUNEL-positive cells in the spleen. TUNEL staining from a representative HeV-infected bat (60 hpi) showed no increase in the number of TUNEL-positive cells in the (E) spleen and/or (F) kidney compared to the control. Scale bar is $100 \mu \mathrm{m}$ in all panels.

the mechanisms by which bats control viral replication will be invaluable to the field of emerging infectious diseases. In addition, system-wide analysis of human responses to infection only provides a picture of the pathways affected during a pathogenic infection. Ideally we would like to have a system-wide comparison between a pathogenic and nonpathogenic response to the same infectious agent. With this in mind, we compared the transcriptome and proteome 
responses of immortalized kidney cells from human and bat following $\mathrm{HeV}$ infection using our recently developed integrated systems-wide approach.

The PaKiT03 and HEK293T cells examined in this study responded very differently to $\mathrm{HeV}$. Even when only high confidence orthologous transcripts are considered, the transcriptomic and proteomic response was significantly different - despite the similar expression profiles of orthologs at 0 hpi. Human HEK293T cells appeared to show little response at $8 \mathrm{hpi}$, followed by a largely suppressive response at $24 \mathrm{hpi}$. PaKiT03 cells, however, demonstrated a strong activation of several immune pathways at 8 and $24 \mathrm{hpi}$, followed by the induction of extrinsic apoptosis at $24 \mathrm{hpi}$ and beyond.

To our knowledge, this is the first study to examine programmed cell death within a bat species. Apoptosis plays an integral part of the host-pathogen interaction. Induction of the innate immune response often leads to the activation of apoptotic processes, typically through members of the TNF family. We have demonstrated that, following $\mathrm{HeV}$ infection, PaKiT03 cells up-regulated components of the TRAILmediated apoptosis pathway. By contrast, the HEK293T cells appeared to either down-regulate a number of proapoptotic proteins or up-regulate anti-apoptotic components. Induction of TRAIL-mediated apoptosis following viral infection of human cells has been reported previously. In most cases, viral infection induces the expression of the death receptors DR4 and DR5, which in turn sensitizes the cells to TRAIL-mediated apoptosis [24,25]. Infection of human cells with hepatitis $C$ and mammalian reoviruses induces mRNA and protein expression of the functional TRAIL receptors DR4 and DR5 [24,26]. Paramyxoviruses such as human respiratory syncytial virus have also been shown to induce expression of DR5 and DR4 in human lung cells [25]. In the PaKiT03 cells, we observed a greater than two-fold increase in TNFRSF10A/DR4 mRNA expression at $24 \mathrm{hpi}$. Interrogation of the $P$. alecto genome along with our de novo assembled transcriptome suggested that, like mice, bats contain only one functional death receptor. TNFRSF10A/DR4 may therefore act as the sole functional TRAIL receptor in bats.

The expression of TRAIL is regulated through NF- $\mathrm{kB}$ transcription factors [27] in response to viral infection. Viruses such as measles and respiratory syncytial virus induce functional TRAIL expression in human cells [25,28]. Here, we observed a significant increase in TRAIL protein expression at $24 \mathrm{hpi}$ in the bat cells. The activation of NF$\mathrm{KB}$ pathways within the bat cells, including the upregulation of CD40, NFKB2 and RelB transcripts, may have contributed to the increased TRAIL protein expression. Surprisingly, we observed no increase in TRAIL mRNA at $8,24,48$ or 72 hpi within the PaKiT03. This finding suggests that the regulation of TRAIL protein expression possibly occurs post-transcriptionally. By using previously collected lung and kidney tissue samples from $\mathrm{HeV}$-infected bats, we examined the expression of TRAIL and CD40 mRNA in the context of an in vivo infection. While no increase in CD40 mRNA expression was observed in either kidney or lung, we found an approximately five-fold up-regulation of TRAIL mRNA in bat lungs infected with $\mathrm{HeV}$ for $60 \mathrm{~h}$. Previous, unpublished data from this trial demonstrated that the $\mathrm{HeV}$ antigen could only be detected in the lung tissue at $60 \mathrm{hpi}$ through immunohistochemistry ( $M$. Baker, unpublished data). The coordinated up-regulation of TRAIL mRNA at the site of $\mathrm{HeV}$ infection within the natural host highlights the importance of TRAIL in $\mathrm{HeV}$ infection in vivo.

The interaction between TRAIL and its functional receptors is crucial for inducing apoptotic cell death through caspase 8 [29]. The treatment of cells with human recombinant TRAIL and subsequent $\mathrm{HeV}$ infection demonstrated that $\mathrm{HeV}$ sensitizes PaKiT03 cells to TRAIL-mediated apoptosis at $24 \mathrm{hpi}$. Presumably, this 'sensitivity' is achieved through the increased expression of TNFRSF10A/DR4 as discussed above. A similar observation was made by Clarke et al. [24], where reovirus infection increased the sensitivity of human cells to apoptosis by increasing the expression of DR4 and DR5. In the present study, HEK293T cells showed no increased susceptibility to TRAIL-mediated apoptosis following $\mathrm{HeV}$ infection. Importantly, we showed that this finding is not a general feature of human HEK293T cells. Indeed, when we treated these cells with human recombinant TRAIL and infected them with NBV, we saw an increase in TRAIL-mediated apoptosis (evidenced by increased caspase $3 / 7$ activity). We can therefore conclude that the HEK293T cells are indeed capable of TRAILinduced cell death, but for unclear reasons this process does not occur in response to $\mathrm{HeV}$.

Pro-apoptotic components down-stream of the death receptors were also induced in the bat cells, including $B B C 3, B I R C 3$ and caspase 13 . The latter, also known as evolutionarily related interleukin $1 \beta$ converting enzyme, was originally discovered in humans [30], but was later shown to be of bovine origin and is the likely ortholog of human caspase 4 [31]. In the present study, a transcript resembling caspase 13 was induced at 8 and 24 hpi in the bat cells. Previous studies have shown that over-expression of caspase 13 in human HEK293 and MCF7 cells successfully induces apoptosis [30]. Furthermore caspase 13 appeared to be activated by caspase 8 [30], suggesting this enzyme may play a role in death receptor-mediated apoptosis pathways, such as TRAIL.

In principle, the ability of PaKiT03 cells to induce apoptotic cell death following $\mathrm{HeV}$ infection could provide a mechanism by which cells can effectively inhibit or subdue viral replication. To determine whether this phenotype was representative of all bat cells we examined $\mathrm{HeV}$ - 
induced apoptosis across three more bat (derived from $P$. alecto) and three more human cell lines. The apoptotic response varied widely between the cell lines. Strong caspase 3/7 responses were observed in HEF and HeLa cells at 24 hpi. The bat fetus cells (PaFeB5) also showed a strong caspase $3 / 7$ response, while the brain (PaBrT03) and lung (PaLuT02) cells were less responsive. While these findings demonstrate that $\mathrm{HeV}$-induced apoptosis is not specific to bat cells, they do highlight the importance of programmed cell death in viral infection more broadly. In agreement, a previous study found NiV partially activates and induces apoptotic cell death of human dendritic cells through upregulation of caspase 3 and down-regulation of bcl2 [32].

Considering the induction of apoptosis in PaKiT03 cells in vitro, along with the up-regulation of TRAIL mRNA in $\mathrm{HeV}$-infected bat lung tissues, we attempted to examine the apoptotic response of bats to $\mathrm{HeV}$ in vivo. TUNEL staining of formalin-fixed paraffinembedded tissue sections revealed no increase in the proportion of apoptotic cells in either the kidney or spleen of $\mathrm{HeV}$-infected bats compared to uninfected controls. In fact, very few apoptotic cells were observed in the kidney in any sample, while numerous apoptotic cells were found in the spleen. Unfortunately, the lung sections (where we might have expected to see the most striking differences) from this trial were not suitable for TUNEL staining, due to artifacts created during euthanasia. While this experiment could not definitively demonstrate $\mathrm{HeV}$-induced apoptosis in vivo in bats, future examination of tissues from other $\mathrm{HeV}$ model species such as ferret and mice - where a more pronounced viral load is achieved - may help clarify the influence of apoptosis in vivo.

We cannot exclude the possibility that the subtle differences in viral transcription between the bat and human cells are influencing the host response. Viruses utilize a number of strategies for counteracting immune and apoptotic signals. Previous studies have demonstrated that paramyxoviruses can use the accessory proteins from the $\mathrm{P} / \mathrm{V} / \mathrm{C}$ gene to inhibit apoptosis [33,34]. The $\mathrm{V}$ protein of mumps virus is also known to block IFN expression [35]. When we compared the $\mathrm{HeV}$ transcription profile between the cell lines, there appeared to be fewer transcripts for the $\mathrm{P} / \mathrm{V} / \mathrm{W}$ region in human cells compare to bat cells. Considering the important role of the $\mathrm{V}$ protein in immune evasion, this transcriptional variation may influence the host response, particularly in regard to IFN production. Phenotypically, we also observed differences in the cytopathic effect between human and bat cells following $\mathrm{HeV}$ infection at $24 \mathrm{hpi}$. Extensive syncytia were observed in the human but not bat cells. Cell fusion and syncytia are mediated by the HeV-F protein. It is therefore interesting to note that we observed higher abundance of $\mathrm{F}$ transcript in the human cells compared to bat cells. Differences in the abundance of $\mathrm{F}$ transcript may therefore influence the difference in cytopathic effect observed.

The induction of IFN- $\beta 1$ and down-stream ISGs in human cells was unexpected. A number of transfection studies have demonstrated that the henipavirus $\mathrm{P}$ gene products (P, V, W and C) can inhibit both the IFN induction and IFN signaling pathways in human cell lines (including HEK293T and 2fTGH) [36,37]. More recent studies on HEK293T cells, however, have shown that, while productive henipavirus infection can effectively block IFN production after 3 hpi, it only partially blocks IFN signaling, as measured by the expression of ISGs at $24 \mathrm{hpi}$ [38]. Similar studies on a range of $P$. alecto cell types, including lung (PaLuT02), primary kidney (PaKi) and fetus (PaFe and PaFeT) [39], demonstrated that both IFN production and signaling were blocked by henipavirus infection at 3 and 24 hpi, respectively [40]. By contrast, our findings demonstrate that in human HEK293T cells IFN production and signaling occur at $24 \mathrm{hpi}$, thus suggesting the host is capable of overcoming the antagonistic effects of $\mathrm{HeV}$ at this later time point. It is worth noting that interferon regulatory factor 3 (IRF3), an important transcription factor responsible for IFN- $\beta$ induction, was down-regulated at the protein level at $24 \mathrm{hpi}$ in human cells. This finding is somewhat at odds with the induction of IFN- $\beta 1$ at 24 hpi in human cells. NiV has been shown to block IFN production through the accessory $\mathrm{V}$ and W proteins by targeting IRF3 [41]. While $\mathrm{HeV}$ may use a similar mechanism, a two-fold reduction in IRF3, as observed in this study, does not appear sufficient to completely abolish IFN production in human cells. IFN antiviral response is also mediated to some degree by the Ras/MAPK pathways. Studies of persistent Ebola virus (Mayinga strain) infection of bat lung fibroblasts found that activation of the Ras/MAPK pathway, which antagonizes the IFN response, was required for productive infection [13]. In the present study, we found many of the downregulated transcripts/proteins in the human cells were mapped to MAPK pathways. Inactivation of these pathways may have contributed to the increased IFN response observed in human cells at $24 \mathrm{hpi}$.

It should be acknowledged that this study, although comprehensive, examined only one cell type from both bats and humans. The major differences in response between the bat and human cells may to some degree simply reflect differences in cell type. In any mammal, it is highly likely that not all cell types will respond to viral infection in a similar fashion. Furthermore, mechanisms deduced from cell lines may not reflect what occurs in vivo. With this in mind, we stress that caution must be taken when extending conclusions from in vitro studies (such as ours) to other cell types, and more generally in vivo models. However, in vitro studies provide significant insight into host mechanisms at the molecular level, ultimately generating numerous hypotheses that can be 
investigated within appropriate models in vivo. Indeed, in vitro studies are necessary (at least in the first instance) when examining a highly pathogenic virus (such as $\mathrm{HeV}$ ) that requires high levels of bio-containment.

Turning to the many novel genes and proteins identified, that we uncovered a great many new genes that are also positively identified by liquid chromatography tandem mass spectrometry (LC-MS/MS) re-enforces the power of our PIT-based approach to comprehensively identify, annotate and quantitate changes in human and non-model host species. This is the first time a single virus has been comprehensively analyzed by RNAseq and high-throughput quantitative proteomics in cells lines from two species. For the first time, we can compare at a systems level, the response between a resistant reservoir host (bat) and a susceptible spillover host (human). By identifying the crucial cellular pathways whose differential activation correlates with outcome, we open new opportunities for therapeutic intervention, through testing the many pathway-specific licensed drugs already available. Moreover, our approach enables side-by-side comparison of any mammal with humans to determine how similar or divergent two species are when they are infected with a virus or exposed to a drug (or both). In principle, this could guide better selection of animal models and improve our ability to predict how humans will respond to treatments developed in animal models. Finally, our dataset provides transcriptomic and proteomic evidence of approximately 5,900 genes and proteins. This includes evidence for nearly several hundred genes and proteins not listed in the UniProt dataset for $P$. alecto. This represents a major leap forward in our understanding of the genetic content of bats - significant natural hosts of current and potentially future zoonotic agents.

\section{Conclusion}

By application of PIT analysis, we have shown that PaKiT03 and HEK293T cells respond significantly differently to $\mathrm{HeV}$ at both the mRNA and protein level. Human HEK293T cells demonstrated little early response followed by a global suppression of mRNA and protein abundance. Bat PaKiT03 cells, by contrast, demonstrated a robust innate immune response, which led to the execution of extrinsic TRAIL apoptosis pathways. At 48 and 72 hpi, PaKiT03 cells, but not HEK293T cells, demonstrated a significant increase in apoptotic cell death. However, when further cell lines from bats and humans were examined, we found that at least some human lines (HEF and HeLa) were susceptible to $\mathrm{HeV}$-mediated apoptosis. Moreover, examination of tissue samples from $\mathrm{HeV}$-infected bats failed to reveal widespread apoptosis at least for the spleen and kidneys. Notwithstanding this, we have shown how to compare the response of two distinct species to the same infectious agent using RNAseq and high-throughput quantitative proteomics. We have used this analysis to highlight the potential to identify and test pathways that in principle could have a significant bearing on the outcome of disease. We believe this approach will enable the identification and investigation of pathways that contribute at a molecular level to the devastating differential pathogenicity of zoonotic bat viruses when they infect humans.

\section{Methods}

\section{Cell culture and SILAC}

The $P$. alecto immortalized kidney derived cell line PaKiT03 [39] and the human immortalized embryonic kidney cell line HEK293T were predominantly used in this study. Cells were cultured in either Dulbecco's modified Eagle's medium (DMEM)/F12 (PaKiT03) or DMEM (HEK293T) media (Pierce, Rockford, USA) containing 10\% (v/v) dialyzed fetal calf serum (FCS), and $10 \mathrm{mM}$ HEPES. To achieve isotope incorporation cells were cultured in either ${ }^{12} \mathrm{C} /{ }^{14} \mathrm{~N}$ lysine and arginine (designated 'light'), ${ }^{13} \mathrm{C} /{ }^{14} \mathrm{~N}$ lysine and arginine (designated 'mid') or ${ }^{13} \mathrm{C} /{ }^{15} \mathrm{~N}$ lysine and arginine (designated 'heavy'). Cells were cultured for at least 10 doublings.

Additional bat cell lines utilized in this study included P. alecto immortalized brain (PaBrT03), fetus (PaFeB5) and lung (PaLuT02) cells [39]. All bat cells were cultured in DMEM/F12 containing 10\% FCS. Additional human cells utilized in this study include A549, HEF and HeLa cells. The A549 and HEF cells were cultured in DMEM containing 10\% FCS. HeLa cells were cultured in Eagle's minimal essential medium containing 10\% FCS.

\section{Virus infection for SILAC cells}

Approximately $2 \times 10^{7}$ PaKiT03 and HEK293T cells were either mock infected (light), or infected with $\mathrm{HeV} /$ Australia/Horse/1994/Hendra strain for $8 \mathrm{~h}$ (mid) or $24 \mathrm{~h}$ (heavy) at an MOI of 10. The experiment was conducted in duplicate in $775 \mathrm{~cm}^{2}$ flasks. Cells were harvested by trypsinization and split in two. One half was resuspended in 2\% SDS-NuPAGE reducing buffer (Life Technologies, Carlsbad, USA) and boiled for $5 \mathrm{~min}$ at $100^{\circ} \mathrm{C}$. The remainder was resuspended in RLT buffer (Qiagen, Limburg, Netherlands) containing 1\% $\beta$ mecaptoethanol.

\section{Immunofluorescence microscopy}

SILAC-labeled PaKiT03 and HEK293T cells were seeded onto $13 \mathrm{~mm}$ diameter coverslips in 24-well plates. Cells were either mock (light cells) or infected for $8 \mathrm{~h}$ (mid) or $24 \mathrm{~h}$ (heavy) with $\mathrm{HeV} /$ Australia/Horse/1994/Hendra strain at an MOI of 10. Media was removed and cells were fixed in $4 \%$ paraformaldehyde $(\mathrm{w} / \mathrm{v})$ in phosphate-buffered saline (PBS) for $1 \mathrm{~h}$. Coverslips were then washed three 
times in PBS, permeabilized with $0.1 \%(\mathrm{w} / \mathrm{v})$ Triton X-100 for $10 \mathrm{~min}$, blocked with $0.5 \%(\mathrm{w} / \mathrm{v})$ bovine serum albumin (BSA) for $30 \mathrm{~min}$, and incubated with anti-N protein (Australian Animal Health Laboratory) diluted 1:2,000 in $0.5 \%(\mathrm{w} / \mathrm{v})$ BSA for $1 \mathrm{~h}$. Cells were washed three times with PBS and treated with a Alexa 488-labeled goat anti-rabbit antibody diluted 1:200 (Life Technologies) in 0.5\% (w/v) BSA for $1 \mathrm{~h}$. Finally, cells were washed twice with PBS, once with $\mathrm{H}_{2} \mathrm{O}$, and stained with 4',6-diamidino-2-phenylindole dihydrochloride for $10 \mathrm{~min}$. Coverslips were washed in PBS, mounted in Vectashield (Vector Labs, Burlingame, USA) and imaged with a Leica SP5 confocal microscope.

\section{RNA isolation and sequencing}

Total RNA was isolated from cells in RLT buffer using the RNAeasy kit (Qiagen) with DNase I treatment as per the manufacturer's instructions. The quality and quantity of RNA was assessed for all samples using a Bioanalyser (Agilent, Santa Carla, USA). mRNA was sequenced as 100 base pair paired-end reads across a single lane on a HiSeq 2000 (Illumina, San Diego, USA). Resulting reads were trimmed for adapters and quality assessed using FastQC. Reads have been deposited in the NCBI Sequence Read Archive and assigned the accession [SRP044809].

\section{Gene Ontology and KEGG enrichment}

Official gene identifications (IDs) were retrieved for all significantly differentially expressed transcripts and proteins. Gene IDs for transcripts and proteins that were significantly up-regulated at one or more time points were combined into a single list. A list of significantly down-regulated gene IDs was also compiled. Biological process GO enrichment was performed separately on the up- and down-regulated transcript and protein lists using the unranked target and background analysis in GOrilla [42]. Background gene lists were compiled by retrieving all gene IDs from either the human (hg19) or P. alecto genome. Enriched GO categories were compiled into Excel spreadsheets and visualized using REVIGO [43]. The up- and down-regulated transcript and protein lists were also used to interrogate KEGG pathways using the standard KEGG Mapper tool [44].

\section{Transcriptome assemblies and differential expression analysis}

Raw reads from each species were first used to assemble a de novo transcriptome on the University of Bristol high performance computing facility, BlueCrystal, using Trinity $[45,46]$. Next, for each of the samples, reads were independently mapped against the $P$. alecto [GenBank: GCF_000325575.1] or human (hg19 in Ensembl) genome with Bowtie (version 0.12.7) and TopHat (version 1.3.2) [47]. Using the alignment outputs, transcripts were assembled for each sample and merged into a single transcriptome for PaKiT03 and HEK293T using Cufflinks and Cuffmerge (version 1.2.1) [48]. Both Trinity and Cufflinks-assembled transcriptomes were translated as previously described [21] using the 'getorf' facility in EMBOSS and ORFs less than 200 nucleotides were removed.

To understand changes in transcript abundance, reads from each time point were mapped separately against the Trinity-generated transcriptome using Bowtie2 (version 2.1.0). Resulting SAM files were converted to BAM, sorted, indexed, and the number of reads mapping to each Trinity transcript was compiled using SAMtools (version 0.1.18). Differential expression analysis was conducted with DESeq (version 1.14.0). Count data was normalized and pairwise comparisons between time points calculated as described previously [49]. Transcripts with adjusted $P$-values $<0.05$ were considered significant.

\section{Quantitative proteomics}

The three SDS boiled protein samples were combined in a 1:1:1 ratio, separated by SDS-PAGE and analyzed by LC-MS/MS. The gel lane was cut into 20 slices, and each slice was subjected to in-gel tryptic digestion and the samples processed as described previously [21]. In addition, the samples from PakTi03 cells were run again (another 20 slices) as a technical repeat to maximize our data return for this species.

The raw data files were processed and quantified using MaxQuant and searched against the three databases described below. Peptide precursor mass tolerance was set at $10 \mathrm{ppm}$, and MS/MS tolerance was set at $0.8 \mathrm{Da}$. Search criteria included carbamidomethylation of cysteine $(+57.0214)$ as a fixed modification and oxidation of methionine (+15.9949) and appropriate SILAC labels $\left({ }^{13} \mathrm{C}_{6}\right.$-lysine, ${ }^{13} \mathrm{C}_{6}$ arginine for mid-labeled cells and ${ }^{13} \mathrm{C} 6,{ }^{15} \mathrm{~N}_{2}$-lysine, ${ }^{13} \mathrm{C}_{6},{ }^{15}$ $\mathrm{N}_{4}$-arginine for heavy-labeled cells as variable modifications). Searches were performed with full tryptic digestion, and a maximum of two missed cleavages was allowed. The reverse database search option was enabled, and all peptide data were filtered to satisfy a false discovery rate of $1 \%$. All raw proteomics data has been deposited into the PRIDE (PRoteomics IDEntifications) database and assigned the accession number [PXD001165].

The lists of proteins derived from a Trinity- or Cufflinks-based assembly of mRNA were searched sequentially on their own to determine which ORFs might be real (that is, at least one peptide identified). From this analysis we obtained a short list of ORFs that had been identified and combined these with the official UniProt lists to create a combined list of proteins from UniProt, from the Trinity transcripts and from the Cufflinksassembled transcripts. To identify the maximum number of peptides, mass spectra were searched against a combination of three protein databases: UniProt-derived lists of proteins for $\mathrm{HeV}$ and either $H$. sapiens or $P$. alecto; ORFs identified as real from the Cufflinks-derived mRNA; and 
ORFs identified as real from the de novo assembled Trinity-derived mRNA. Peptides were assigned by MaxQuant into 'proteinGroups' that contain one or more individual protein sequences (Additional files 7 and 8). In most cases peptides were assigned to a proteinGroup that contained a protein sequence from all three databases (Additional file 9). For the PaKiT03 cells the greatest number of proteinGroups was identified against the Trinity de novo assembled transcript database. By contrast, for the HEK293T cells the greatest number of proteinGroups was identified against the UniProt human proteins list (Additional file 9). Differential expression of the proteinGroups was calculated from the combined peptide abundance ratio of mid to light ( $8 \mathrm{hpi}: 0 \mathrm{hpi})$ and heavy to light (24 hpi:0 hpi). A threshold of $\geq$ two-fold change was employed. That is, protenGroups with SILAC ratios $\geq 2$ or $\leq 0.5$ were considered significant.

\section{Comparison between human and Pteropus datasets}

To identify orthologs shared between bats and humans we used the Trinity-derived lists of transcripts. ORFs (from start codon to stop codon) were translated in all frames from the Trinity-derived transcripts and ORFs over 66 amino acids long were compiled into a table together with the transcript they came from. This list of ORFs was searched using BLAST to find the closest homolog in the human UniProt list. This process was repeated for both the human and the bat list of transcripts. Finally, for each entry in the human UniProt list of proteins we selected the best match from the Trinity-derived ORFs for each species. In this way we were able to generate a master list of transcripts and ORFs that were the best matches to the human UniProt list of proteins. This approach allowed us to connect orthologous genes and ORFs from the human Trinity-derived transcripts to the $P$. alecto Trinity-derived transcripts and ORFs. We were then able to designate the $P$. alecto genes and proteins with their nearest human homologs, enabling a pathways analysis based on the assumption that all the identified $P$. alecto genes and proteins had direct human homologs (Additional file 4). Alongside this analysis, we examined the list of $P$. alecto Trinity-derived ORFs that had been identified as being real on the basis that at least one peptide from each ORF had been detected by the MaxQuant analysis. In essence, each transcript that generated an ORF identified by MaxQuant was compiled into a list of transcripts, ORF and peptide evidence for each ORF. We then used sequential BLAST searches to identify each ORF by searching against the $P$. alecto proteome and the human proteome (Additional file 10). We separated out any ORF which had a less than $60 \%$ identity to either official proteome list and searched only those against the non-redundant protein lists, again using BLAST (Additional file 11).

\section{Bat tissue samples}

Tissue samples from $P$. alecto were obtained from a previous experimental $\mathrm{HeV}$ infection at the Australian Animal Health Laboratory. Seven age-matched Australian black flying foxes were each intranasally infected with 27,300 Tissue culture infective dose (TCID50)/ml of $\mathrm{HeV}$ (Redlands strain). Two uninfected bats served as controls. Animals were euthanized at $12(n=3), 36(n=2)$ and $60(n=2)$ hpi, and tissues dissected and stored. RNA was extracted (as described above) from lung and kidney for the $36 \mathrm{hpi}, 60 \mathrm{hpi}$ and control bats. Lung, kidney and spleen tissues were formalin fixed and paraffin embedded for all bats. All animal experimentation was approved by the Commonwealth Scientific and Industrial Research Organisation-Australian Animal Health Laboratory Animal Ethics Committee (protocol AEC1558).

\section{Real-time PCR}

Differential gene expression was assessed using reverse transcriptase real-time PCR. Primers were designed using Primer3 (Additional file 12). Reaction parameters were identical for all genes. Briefly, $5 \mu \mathrm{g}$ of total RNA was reverse transcribed using Superscript III (Life Technologies) primed with oligo-dT as per the manufacturer's instructions. Triplicate SYBR green real-time PCR reactions were performed in a $25 \mu \mathrm{l}$ reaction, containing $1 \mathrm{X}$ EXPRESS SYBR green master mix (Life Technologies), $200 \mathrm{nM}$ forward and reverse primer, and $20 \mathrm{ng}$ template. Cycling parameters were $95^{\circ} \mathrm{C}$ for $10 \mathrm{~min}$, then 40 cycles of $95^{\circ} \mathrm{C}$ for $30 \mathrm{~s}, 55^{\circ} \mathrm{C}$ for $30 \mathrm{~s}$ and $72^{\circ} \mathrm{C}$ for $1 \mathrm{~min}$, followed by melt curve analysis. Differential expression was calculated relative to uninfected $\mathrm{T} 0$, normalized to GAPDH using the relative expression software tool REST [50].

\section{Western blotting}

Protein quantity was determined for SDS boiled lysates using the EZQ Protein Quantitation Kit (Life Technologies) following the manufacturer's instructions. For each sample a total of $10 \mu \mathrm{g}$ of protein lysate was subjected to SDS-PAGE and transferred to a polyvinylidene difluoride membrane. Membranes were blocked with 5\% skim milk powder for $1 \mathrm{~h}$. Membranes were washed three times (10 min each) with Tris-buffered saline/Tween 20 (TBST), and then incubated for $1 \mathrm{~h}$ with primary antibody diluted in TBST. The following primary rabbit antibodies were used: CD40 (1:5,000, Thermo Fisher, Waltham, USA), A20 (1:1,000, Cell Signaling, Danvers, USA) and $\beta 2$-tubulin (1:2,000, Cell Signaling). Membranes were washed three times with TBST and then incubated for $1 \mathrm{~h}$ with goat anti-rabbit horseradish peroxidase-conjugated secondary antibody diluted in TBST. Membranes were finally washed twice with TBST, once with TBS and developed with ECL-Plus chemiluminescent substrate (Thermo Fisher) as per the manufacturer's 
instructions. Membranes were scanned at $473 \mathrm{~nm}$ on a Typhon FLA9000 gel imaging scanner (GE Healthcare, Little Chalfont, UK).

\section{Apoptosis and viability assay}

The Caspase-Glo 3/7 Assay (Promega, Madison, USA) was used to determine the activity of caspase $3 / 7$. Cell viability was determined using the CellTiter-Glo Luminescent Cell Viability Assay (Promega). For both assays, approximately, 30,000 cells/well were seeded into white 96-well plates. Cells were infected with $\mathrm{HeV}$ or NBV [22] for 24, 48 and $72 \mathrm{~h}$ at an MOI of approximately 5 . Both assays were performed exactly as per the manufacturer's instructions, in triplicate. TRAIL stimulation with performed using SuperKillerTRAIL (AdipoGen, San Diego, USA) at $500 \mathrm{ng} / \mathrm{ml}$ diluted in media. Oneway analysis of variance was used to compare the caspase 3/7 luminescence over the control.

\section{TUNEL staining}

TUNEL was used to identify apoptotic PaKiT03 and HEK293T cells following viral infection. Approximately $1.5 \times 10^{5}$ cells were seeded onto $15 \mathrm{~mm}$ diameter coverslips in individual wells of a 24-well plate. Cells were either mock or infected for 8,24 or $48 \mathrm{~h}$ with $\mathrm{HeV} / 1994 /$ Australia strain at an MOI of 5 . Media was removed and cells were fixed in $4 \%(\mathrm{w} / \mathrm{v})$ paraformaldehyde in PBS for $1 \mathrm{~h}$. Cells were then gently washed three times in PBS. Staining was performed using a Click-iT TUNEL Alexa Fluor ${ }^{\circ} 488$ kit as per the manufacturer's instructions (Life Technologies). Optional immunofluorescence was performed against the $\mathrm{HeV}-\mathrm{N}$ protein as described above except a secondary Alexa Fluor 568 Goat Anti-Rabbit antibody (Life Technologies) was used.

Colormetric TUNEL staining was performed on Australian black flying fox spleen and kidney tissue sections from a previous unpublished experimental $\mathrm{HeV}$ infection. Following routine histological processing and paraffin embedding, blocks were sectioned at $4 \mu \mathrm{m}$ on to positively charged slides and placed in a $37^{\circ} \mathrm{C}$ oven to dry. Sections were placed in a $60^{\circ} \mathrm{C}$ incubator for $10 \mathrm{~min}$ prior to de-waxing and rehydration through a series of graded alcohols to water. The TUNEL staining for apoptotic cells was carried out as per the manufacturer's instructions for tissue sections using the DeadEnd Colorimetric TUNEL System (Promega) with the exception of a substitution of 3-amino-9-ethylcarbazol for $3,3^{\prime}$-diaminobenzidine as the chromogen. The sections were subsequently counterstained with Lillie-Mayer's hematoxylin to enable visualization of the morphological structure of the tissue.

\section{Additional files}

\begin{abstract}
Additional file 1: List of differentially expressed Trinity transcripts and proteins at 8 and 24 hpi in PaKiT03 (sheet one) and HEK293T cells (sheet two). Top BLAST hit against SwissProt is given.

Additional file 2: Full list of Trinity transcript DEseq and SILAC protein expression statistics in PaKiT03.
\end{abstract}

Additional file 3: Full list of Trinity transcript DEseq and SILAC protein expression statistics in HEK293T.

Additional file 4: Ortholog identification of between human and bat. The most similar ortholog between bat and human is given.

Additional file 5: Enriched GO terms from combined transcript and protein lists from GOrilla analysis.

Additional file 6: Enriched KEGG pathways from combined transcript and protein lists.

Additional file 7: All proteinGroups identified in PaKiT03 cells through interrogation of UniProt, Cufflinks and Trinity proteome databases.

Additional file 8: All proteinGroups identified in HEK293T cells through interrogation of UniProt, Cufflinks and Trinity proteome databases.

Additional file 9: Mass spectra were searched against three protein databases: UniProt ( $H$. sapiens or $P$. alecto), translated Trinity transcriptome and translated Cufflinks transcriptome for (A)

PaKiT03 and (B) HEK293T. The number of proteinGroups identified and shared between the databases is given.

Additional file 10: BLAST analysis of bat Trinity transcripts where one or more peptides were identified. BLAST was used against the $P$. alecto and human proteomes (UniProt).

Additional file 11: BLAST analysis against NCBI non-redundant database of Trinity transcripts that were $<60 \%$ similar to both the $P$. alecto and human proteomes.

Additional file 12: Primer sequences used for this study.

\section{Abbreviations}

BLAST: Basic Local Alignment Search Tool; BSA: bovine serum albumin; DMEM: Dulbecco's modified Eagle's medium; FCS: fetal calf serum; GO: Gene Ontology; HeV: Hendra virus; hpi: hours post infection; IFN: interferon; ISG: interferon-stimulated gene; KEGG: Kyoto Encyclopedia of Genes and Genomes; LC-MS/MS: liquid chromatography tandem mass spectrometry; MOI: multiplicity of infection; NBV: Nelson Bay virus; NF-kB: nuclear factor kappa-light-chain-enhancer of activated B cells; Niv: Nipah virus; ORF: open reading frame; PARP1: poly (ADP-ribose) polymerase 1; PBS:

phosphate-buffered saline; PCR: polymerase chain reaction; PIT: proteomics informed by transcriptomics; SDS-PAGE: sodium dodecyl sulfate polyacrylamide gel electrophoresis; SILAC: stable isotope labeling of amino acid in cell culture; TBST: tris-buffered saline and Tween 20; TNF: tumor necrosis factor; TRAIL: tumor necrosis factor-related apoptosis inducing ligand; TUNEL: terminal deoxynucleotidyl transferase dUTP nick end labeling.

\section{Competing interests}

The authors declare that they have no competing interests.

\section{Authors' contributions}

JWW, MLB, DM and L-FW conceived and designed the study. JWW, BJS, KH, GAM, VB, PZ, RK, ST and LM performed all wet-laboratory experiments, including viral infections, mass spectrometry, PCR and microscopy. All bioinformatic analysis was performed by JWW and DM, with advice from MT. PM and DG assisted and advised on confocal microscopy. JH, JP and JB advised and conducted TUNEL staining. JWW and DM wrote the manuscript. All authors read and approved the final manuscript.

\section{Acknowledgements}

The authors wish to thank Dr Wojtek Michalski, Dr Chris Cowled and Dr Meagan Gillespie from the Commonwealth Scientific and Industrial Research Organisation (CSIRO) for constructive advice on this project and manuscript. 
We also thank Dr Nadine Dudek (Monash University) for assistance with mass spectrometry. The authors would also like to acknowledge Professor Martin Pera (University of Melbourne) and Dr Anna Michalska (University of Melbourne) for providing the HEF cells to LM, and Cathy Williams from CSIRO for providing all other cell lines. The study is supported in part by the CSIRO OCE Science Leader Award (to L-FW), the Australian ARC Future Fellowship (FT110100234, to MLB), and the Singaporean NRF Competitive Research Programme Grant (NRF-CRP10-2012-05; to L-FW). We thank lan Stewart and Callum Wright for help with data analysis on the University of Bristol supercomputer, BlueCrystal.

\section{Author details}

${ }^{1}$ CSIRO Biosecurity Flagship, Australian Animal Health Laboratory, East Geelong, VIC, Australia. ${ }^{2}$ Department of Cellular and Molecular Medicine, School of Medical Sciences, University of Bristol, Bristol, UK. ${ }^{3}$ Program in Emerging Infectious Diseases, Duke-National University of Singapore Graduate Medical School, Singapore, Singapore.

Received: 7 October 2014 Accepted: 6 November 2014

Published online: 15 November 2014

\section{References}

1. Jones KE, Patel NG, Levy MA, Storeygard A, Balk D, Gittleman JL, Daszak P: Global trends in emerging infectious diseases. Nature 2008, 451:990-993.

2. Luis AD, Hayman DTS, O'Shea TJ, Cryan PM, Gilbert AT, Pulliam JRC, Mills JN, Timonin ME, Willis CKR, Cunningham AA, Fooks AR, Rupprecht CE, Wood $J \mathrm{LN}$, Webb CT: A comparison of bats and rodents as reservoirs of zoonotic viruses: are bats special? Proc R Soc Lond B Biol Sci 2013, 280:20122753.

3. O'Sullivan JD, Allworth AM, Paterson DL, Snow TM, Boots R, Gleeson L, Gould AR, Hyatt AD, Bradfield J: Fatal encephalitis due to novel paramyxovirus transmitted from horses. Lancet 1997, 349:93-95.

4. Selvey LA, Wells RM, Mccormack JG, Ansford AJ, Murray K, Rogers RJ, Lavercombe PS, Selleck P, Sheridan JW: Infection of humans and horses by a newly described morbillivirus. Med J Aust 1995, 162:642-645.

5. Chua KB, Goh KJ, Wong KT, Kamarulzaman A, Tan PSK, Ksiazek TG, Zaki SR, Paul G, Lam SK, Tan CT: Fatal encephalitis due to Nipah virus among pig-farmers in Malaysia. Lancet 1999, 354:1257-1259.

6. Lau SKP, Woo PCY, Li KSM, Huang Y, Tsoi HW, Wong BHL, Wong SSY, Leung $S Y$, Chan KH, Yuen KY: Severe acute respiratory syndrome coronavirus-like virus in Chinese horseshoe bats. Proc Natl Acad Sci U S A 2005, 102:14040-14045.

7. Li WD, Shi ZL, Yu M, Ren WZ, Smith C, Epstein JH, Wang HZ, Crameri G, Hu ZH, Zhang HJ, Zhang JH, McEachern J, Field H, Daszak P, Eaton BT, Zhang SY, Wang LF: Bats are natural reservoirs of SARS-like coronaviruses. science 2005, 310:676-679.

8. Leroy EM, Kumulungui B, Pourrut $X$, Rouquet $P$, Hassanin A, Yaba P, Delicat A, Paweska JT, Gonzalez JP, Swanepoel R: Fruit bats as reservoirs of Ebola virus. Nature 2005, 438:575-576.

9. Corman VM, Ithete NL, Richards LR, Schoeman MC, Preiser W, Drosten C, Drexler JF: Rooting the phylogenetic tree of MERS-Coronavirus by characterization of a conspecific virus from an African Bat. J Virol 2014. in press.

10. Ithete NL, Stoffberg S, Corman VM, Cottontail VM, Richards LR, Schoeman MC, Drosten C, Drexler JF, Preiser W: Close relative of human Middle East respiratory syndrome coronavirus in bat, South Africa. Emerg Infect Dis 2013, 19:1697-1699

11. Field H, McCall B, Barrett J: Australian bat lyssavirus infection in a captive juvenile black flying fox. Emerg Infect Dis 1999, 5:438-440.

12. McColl KA, Chamberlain T, Lunt RA, Newberry KM, Middleton D, Westbury HA: Pathogenesis studies with Australian bat lyssavirus in grey-headed flying foxes (Pteropus poliocephalus). Aust Vet J 2002, 80:636-641.

13. Strong JE, Wong G, Jones SE, Grolla A, Theriault S, Kobinger GP, Feldmann $H$ : Stimulation of Ebola virus production from persistent infection through activation of the Ras/MAPK pathway. Proc Natl Acad Sci U S A 2008, 105:17982-17987.

14. Zhang GJ, Cowled C, Shi ZL, Huang ZY, Bishop-Lilly KA, Fang XD, Wynne JW, Xiong ZQ, Baker ML, Zhao W, Tachedjian M, Zhu YB, Zhou P, Jiang XT, Ng J, Yang L, Wu LJ, Xiao J, Feng Y, Chen YX, Sun XQ, Zhang Y, Marsh GA, Crameri G, Broder CC, Frey KG, Wang LF, Wang J: Comparative analysis of bat genomes provides insight into the evolution of flight and immunity. Science 2013, 339:456-460.

15. Murray K, Selleck P, Hooper P, Hyatt A, Gould A, Gleeson L, Westbury H, Hiley L, Selvey L, Rodwell B, Ketterer P: A morbillivirus that caused fatal disease in horses and humans. Science 1995, 268:94-97.

16. Lindblad-Toh K, Garber M, Zuk O, Lin MF, Parker BJ, Washietl S, Kheradpour P, Ernst J, Jordan G, Mauceli E, Ward LD, Lowe CB, Holloway AK, Clamp M, Gnerre S, Alfoldi J, Beal K, Chang J, Clawson H, Cuff J, Di Palma F, Fitzgerald S, Flicek P, Guttman M, Hubisz MJ, Jaffe DB, Jungreis I, Kent WJ, Kostka D, Lara $M$, et al: A high-resolution map of human evolutionary constraint using 29 mammals. Nature 2011, 478:476-482.

17. Parker J, Tsagkogeorga G, Cotton JA, Liu Y, Provero P, Stupka E, Rossiter SJ: Genome-wide signatures of convergent evolution in echolocating mammals. Nature 2013, 502:228-231.

18. Seim I, Fang $X D$, Xiong $Z Q$, Lobanov AV, Huang $Z Y$, Ma SM, Feng $Y$, Turanov AA, Zhu YB, Lenz TL, Gerashchenko MV, Fan DD, Yim SH, Yao XM, Jordan D, Xiong YQ, Ma Y, Lyapunov AN, Chen GX, Kulakova OI, Sun YD, Lee SG, Bronson RT, Moskalev AA, Sunyaev SR, Zhang GJ, Krogh A, Wang J, Gladyshev VN: Genome analysis reveals insights into physiology and longevity of the Brandt's bat Myotis brandtii. Nat Commun 2013, 4:2212.

19. Papenfuss AT, Baker ML, Feng ZP, Tachedjian M, Crameri G, Cowled C, Ng J, Janardhana $V$, Field HE, Wang LF: The immune gene repertoire of an important viral reservoir, the Australian black flying fox. BMC Genomics 2012, 13:261.

20. Shaw TI, Srivastava A, Chou WC, Liu L, Hawkinson A, Glenn TC, Adams R, Schountz T: Transcriptome sequencing and annotation for the Jamaican fruit bat (Artibeus jamaicensis). PLoS One 2012, 7:e48472.

21. Evans VC, Barker G, Heesom KJ, Fan J, Bessant C, Matthews DA: De novo derivation of proteomes from transcriptomes for transcript and protein identification. Nat Methods 2012, 9:1207-1211.

22. Gard GP, Marshall ID: Nelson-Bay Virus - novel reovirus. Arch Gesamte Virusforsch 1973, 43:34-42.

23. Voon K, Chua KB, Yu M, Crameri G, Barr JA, Malik Y, Wang LF: Evolutionary relationship of the $\mathrm{L}$ - and $\mathrm{M}$-class genome segments of bat-borne fusogenic orthoreoviruses in Malaysia and Australia. J Gen Virol 2011, 92:2930-2936

24. Clarke P, Meintzer SM, Gibson S, Widmann C, Garrington TP, Johnson GL, Tyler KL: Reovirus-induced apoptosis is mediated by TRAIL. J Virol 2000, 74:8135-8139.

25. Kotelkin A, Prikhod'ko EA, Cohen Jl, Collins PL, Bukreyev A: Respiratory syncytial virus infection sensitizes cells to apoptosis mediated by tumor necrosis factor-related apoptosis-inducing ligand. J Virol 2003, 77:9156-9172

26. Deng ZF, Yan HJ, Hu JJ, Zhang SW, Peng P, Liu QZ, Guo DY: Hepatitis C virus sensitizes host cells to TRAIL-induced apoptosis by up-regulating DR4 and DR5 via a MEK1-dependent pathway. PLoS One 2012, 7:e37700

27. Baetu TM, Kwon H, Sharma S, Grandvaux N, Hiscott J: Disruption of NFkappa B signaling reveals a novel role for NF-kappa B in the regulation of TNF-related apoptosis-inducing ligand expression. J Immunol 2001, 167:3164-3173.

28. Vidalain PO, Azocar O, Lamouille B, Astier A, Rabourdin-Combe C, ServetDelprat C: Measles virus induces functional TRAIL production by human dendritic cells. J Virol 2000, 74:556-559.

29. Kassis R, Larrous F, Estaquier J, Bourhy H: Lyssavirus matrix protein induces a poptosis by a TRAIL-dependent mechanism involving caspase-8 activation. J Virol 2004, 78:6543-6555.

30. Humke EW, Ni J, Dixit VM: ERICE, a novel FLICE-activatable caspase. J Biol Chem 1998, 273:15702-15707.

31. Koenig U, Eckhart L, Tschachler E: Evidence that caspase-13 is not a human but a bovine gene. Biochem Biophys Res Commun 2001, 285:1150-1154.

32. Gupta M, Lo MK, Spiropoulou CF: Activation and cell death in human dendritic cells infected with Nipah virus. Virology 2013, 441:49-56.

33. Chambers R, Takimoto T: Antagonism of innate immunity by paramyxovirus accessory proteins. Viruses-Basel 2009, 1:574-593.

34. Sun M, Rothermel TA, Shuman L, Aligo JA, Xu SB, Lin Y, Lamb RA, He B: Conserved cysteine-rich domain of paramyxovirus simian virus $5 \mathrm{~V}$ protein plays an important role in blocking apoptosis. J Virol 2004 78:5068-5078.

35. Yang ZH: PAML: a program package for phylogenetic analysis by maximum likelihood. Comput Appl Biosci 1997, 13:555-556. 
36. Rodriguez JJ, Horvath CM: Host evasion by emerging paramyxoviruses: Hendra virus and Nipah virus $\mathrm{V}$ proteins inhibit interferon signaling. Viral Immunol 2004, 17:210-219.

37. Rodriguez JJ, Parisien JP, Horvath CM: Nipah virus V protein evades alpha and gamma interferons by preventing STAT1 and STAT2 activation and nuclear accumulation. J Virol 2002, 76:11476-11483.

38. Virtue ER, Marsh GA, Wang LF: Interferon signaling remains functional during henipavirus infection of human cell lines. J Virol 2011, 85:4031-4034

39. Crameri G, Todd S, Grimley S, McEachern JA, Marsh GA, Smith C, Tachedjian M, De Jong C, Virtue ER, Yu M, Bulach D, Liu JP, Michalski WP, Middleton D, Field HE, Wang LF: Establishment, immortalisation and characterisation of Pteropid bat cell lines. PLoS One 2009, 4:e8266.

40. Virtue ER, Marsh GA, Baker ML, Wang LF: Interferon production and signaling pathways are antagonized during henipavirus infection of fruit bat cell lines. PLoS One 2011, 6:e22488.

41. Shaw ML, Cardenas WB, Zamarin D, Palese P, Basler CF: Nuclear localization of the Nipah virus $W$ protein allows for inhibition of both virus- and tolllike receptor 3-triggered signaling pathways. J Virol 2005, 79:6078-6088.

42. Eden E, Navon R, Steinfeld I, Lipson D, Yakhini Z: GOrilla: a tool for discovery and visualization of enriched GO terms in ranked gene lists. BMC Bioinformatics 2009, 10:48.

43. Supek F, Bosnjak M, Skunca N, Smuc T: REVIGO summarizes and visualizes long lists of gene ontology terms. PLoS One 2011, 6:e21800.

44. Kanehisa M, Goto S: KEGG: Kyoto Encyclopedia of Genes and Genomes. Nucleic Acids Res 2000, 28:27-30.

45. Grabherr MG, Haas BJ, Yassour M, Levin JZ, Thompson DA, Amit I, Adiconis X, Fan L, Raychowdhury R, Zeng QD, Chen ZH, Mauceli E, Hacohen N, Gnirke A, Rhind N, di Palma F, Birren BW, Nusbaum C, Lindblad-Toh K, Friedman N, Regev A: Full-length transcriptome assembly from RNA-Seq data without a reference genome. Nat Biotechnol 2011 29:644-652.

46. Haas BJ, Papanicolaou A, Yassour M, Grabherr M, Blood PD, Bowden J, Couger MB, Eccles D, Li B, Lieber M, MacManes MD, Ott M, Orvis J, Pochet N, Strozzi F, Weeks N, Westerman R, William T, Dewey CN, Henschel R, Leduc RD, Friedman N, Regev A: De novo transcript sequence reconstruction from RNA-seq using the Trinity platform for reference generation and analysis. Nat Protoc 2013, 8:1494-1512.

47. Trapnell C, Pachter L, Salzberg SL: TopHat: discovering splice junctions with RNA-Seq. Bioinformatics 2009, 25:1105-1111.

48. Trapnell C, Roberts A, Goff L, Pertea G, Kim D, Kelley DR, Pimentel H, Salzberg SL, Rinn JL, Pachter L: Differential gene and transcript expression analysis of RNA-seq experiments with TopHat and Cufflinks. Nat Protoc 2012, 7:562-578.

49. Anders $S$, Huber W: Differential expression analysis for sequence count data. Genome Biol 2010, 11:R106.

50. Pfaffl MW, Horgan GW, Dempfle L: Relative expression software tool (REST) for group-wise comparison and statistical analysis of relative expression results in real-time PCR. Nucleic Acids Res 2002, 30:e36.

doi:10.1186/s13059-014-0532-x

Cite this article as: Wynne et al:: Proteomics informed by transcriptomics reveals Hendra virus sensitizes bat cells to TRAIL-mediated apoptosis. Genome Biology 2014 15:532.

\section{Submit your next manuscript to BioMed Central and take full advantage of:}

- Convenient online submission

- Thorough peer review

- No space constraints or color figure charges

- Immediate publication on acceptance

- Inclusion in PubMed, CAS, Scopus and Google Scholar

- Research which is freely available for redistribution

Submit your manuscript at www.biomedcentral.com/submit
C Biomed Central 